\title{
Epigenetic regulation of innate immune memory in microglia
}

2

3 Xiaoming Zhang ${ }^{1, \#}$, Laura Kracht ${ }^{1, \#}$, Antonio M. Lerario ${ }^{2, \#}$, Marissa L. Dubbelaar ${ }^{1}$, Nieske

4 Brouwer $^{1}$, Evelyn M. Wesseling ${ }^{1}$, Erik W.G.M. Boddeke ${ }^{1,3}$, Bart J.L. Eggen ${ }^{1, *, @, ~ S u s a n n e ~ M . ~}$

5 Kooistra1,*,@

6

$7 \quad{ }^{1}$ Department of Biomedical Sciences of Cells \& Systems, Section Molecular Neurobiology,

8 University Medical Center Groningen, University of Groningen, Antonius Deusinglaan 1, 9713

9 AV Groningen, The Netherlands.

10 2Department of Internal Medicine, Division of Metabolism, Endocrinology, and Diabetes, 11 University of Michigan, Ann Arbor, Michigan, USA.

$12{ }^{3}$ Center for Healthy Aging, Department of Cellular and Molecular Medicine, University of

13 Copenhagen, Blegdamsvej 3B, 2200 Copenhagen, Denmark.

14

15 "shared first authors

16 *shared senior authors

17 @correspondence: b.j.l.eggen@umcg.nl, s.m.kooistra@umcg.nl, Tel +31-50-3616432 


\section{Abstract}

20 Microglia are the tissue-resident macrophages of the CNS. They originate in the yolk sac, 21 colonize the CNS during embryonic development and form a self-sustaining population with limited turnover. A consequence of their relative slow turnover is that microglia can serve as a long-term memory for inflammatory or neurodegenerative events. We characterized the epigenomes and transcriptomes of microglia exposed to different stimuli; an endotoxin challenge (LPS) and genotoxic stress (DNA repair deficiency-induced accelerated aging). Whereas the enrichment of permissive epigenetic marks at enhancer regions explains training (hyperresponsiveness) of primed microglia to LPS challenge, the tolerized response of microglia seems to be regulated by loss of permissive epigenetic marks. Here, we identify that inflammatory stimuli and accelerated aging because of genotoxic stress activate distinct gene networks. These gene networks and associated biological processes are partially overlapping, which is likely driven by specific transcription factor networks, resulting in altered epigenetic signatures and distinct functional (desensitized vs. primed) microglia phenotypes.

\section{Keywords}

35 Microglia, chromatin, tolerance, priming, innate immunity, neuroinflammation 


\section{Introduction}

Microglia are of myeloid lineage and are long-lived tissue-resident macrophages of the central nervous system (CNS) parenchyma (Prinz et al., 2017).

Macrophages possess innate immune memory (IMM). IMM describes the concept that macrophages, after experiencing a primary 'priming' or 'desensitizing' stimulus, react with a stronger (immune training) or weaker (immune tolerance) immune response to a subsequent stimulus (Neher \& Cunningham, 2019; Netea et al., 2011). IMM was discovered and has been extensively described in blood-derived monocytes/macrophages (Biswas \& Lopez-Collazo, 2009; Kleinnijenhuis et al., 2012; Netea et al., 2016; Novakovic et al., 2016; Saeed et al., 2014).

Similar functional states have been described for microglia in mouse models (Eggen et al., 2013; Neher \& Cunningham, 2019). Primed microglia can be elicited in mouse models of prion disease (Cunningham et al., 2005), neurodegeneration (Norden et al., 2015; Wendeln et al., 2018), natural aging (Sierra et al., 2007) and neuronal genotoxic stress-induced accelerated aging (D. D. A. Raj et al., 2014). When these mice experienced a peripheral lipopolysaccharide (LPS) challenge, microglia exhibited an excessive immune response manifested by increased expression of proinflammatory cytokines, called microglia training (Cunningham et al., 2005; D. D. A. Raj et al., 2014; Sierra et al., 2007; Wendeln et al., 2018). Oppositely, mouse microglia can be desensitized with LPS (Longhi et al., 2011; Schaafsma et al., 2015; Schaafsma et al., 2017). After a secondary challenge, in the form of LPS (Schaafsma et al., 2015; Schaafsma et al., 2017), traumatic brain injury (Longhi et al., 2011) or cerebral ischemia (J. T. Yu et al., 2010), microglia display immune tolerance which is defined by a reduced immune response.

Microglia are implicated in CNS development, and neurodevelopmental and neurodegenerative diseases (Hammond et al., 2019; Holtman et al., 2015; Kracht et al., 2020; Li et al., 2019; Matcovitch-Natan et al., 2016; Salter \& Stevens, 2017; Sarlus \& Heneka, 2017). It is especially interesting to investigate microglia IMM in this context. The combination of perturbations like maternal immune activation during vulnerable periods of CNS development together with the occurrence of multiple stimuli over a long period of time is thought to cause neurodevelopmental or neurodegenerative diseases (Knuesel et al., 2014). Microglia are the prime cells that respond to CNS stimuli since they express a wide range of cell surface receptors and adhesion molecules (homeostatic gene signature) through which they can sense those endogenous and exogenous stimuli (Butovsky et al., 2014; Galatro, Holtman, et al., 2017; Gautier et al., 2012; Gosselin et al., 2014, 2017; Hickman et al., 2013; Lavin et al., 2014).

Epidemiologic studies report that infections during specific periods of pregnancy increase the risk for the child to develop neurodevelopmental disorders, like autism or schizophrenia (Estes \& McAllister, 2016). Mouse models of maternal immune activation suggest a role for microglia IMM in this process (Knuesel et al., 2014; Neher \& Cunningham, 2019). Peripheral LPS challenge of pregnant mouse dams caused preconditioning of offspring microglia which 
long-lastingly affects microglia LPS responsiveness in adult offspring and also caused behavioral abnormalities (Schaafsma et al., 2017).

In case of neurodegenerative diseases, genetic risk loci are generally immune-related (Cooper-Knock et al., 2017; T. Raj et al., 2014) and specifically enriched in microglia (Nott et al., 2020). A common gene signature was identified in multiple mouse models of neurodegenerative diseases, aging and priming and encompasses genes such as $\mathrm{Axl}, \mathrm{Clec7a}$ and Mac2 (Holtman et al., 2015). This microglia transcriptional phenotype is orchestrated by the APOE-TREM2 pathway and is associated with altered phagocytic and lysosomal activity, and lipid metabolism (Butovsky \& Weiner, 2018; Holtman et al., 2015; Keren-Shaul et al., 2017; Krasemann et al., 2017). Given the chronic nature of neurodegenerative diseases, it is hypothesized that microglia are trapped in a primed/trained state ultimately leading to neurotoxicity (Block et al., 2007; Haley et al., 2017). This hypothesis was recently confirmed by the observation that induction of priming of microglia in early adulthood caused exacerbation of $A \beta$ pathology later in life, whereas desensitization of microglia diminished $A \beta$ pathology in a mouse model of $A D$ (Wendeln et al., 2018). Current studies suggest microglia priming and tolerance to have neurotoxic (Schaafsma et al., 2017; Wendeln et al., 2018) or neuroprotective (Longhi et al., 2011; Wendeln et al., 2018; J. T. Yu et al., 2010) consequences, respectively. However, these outcomes should not be generalized and the effects of microglia tolerance and priming on neuronal viability need to be elucidated in a context-specific manner (Neher \& Cunningham, 2019).

Both tolerant and trained immunity of peripheral macrophages are long-lasting changes in functionality that are instructed by epigenetic reprogramming (Cheng et al., 2014; El Gazzar et al., 2008; Kleinnijenhuis et al., 2012; Novakovic et al., 2016; Quintin et al., 2012). Though epigenetic programming has been clearly implicated in the segregation of microglia from other tissue resident macrophages in both mouse and human (Gosselin et al., 2014, 2017; Lavin et al., 2014), little is known about the changes in epigenetic signatures in microglia in response to (systemic) immune stimuli or endogenous neuronal damage and how epigenetic memory serves to change subsequent responses. Since microglia are relatively long-lived cells (Füger et al., 2017; Tay et al., 2017), experience of past stimuli is long-lastingly secured in the microglial epigenome and can thus have persistent consequences on microglia functionality and neuronal viability. Several lines of evidence suggest a role for epigenetic regulation of microglia functional states (Cho et al., 2015; Keren-Shaul et al., 2017; Matcovitch-Natan et al., 2016; Matt et al., 2016; Schaafsma et al., 2015; Wendeln et al., 2018; Yeh \& Ikezu, 2019).

To delineate the gene networks and associated epigenetic signatures and transcription factors that underlie functional microglia states of priming and tolerance, we acutely isolated microglia from mice challenged with LPS and from accelerated aging mice and analyzed their transcriptional and chromatin status at a genome-wide level. 


\section{Results}

111 LPS desensitization and accelerated aging result in distinct transcriptional responses 112 in microglia

113 In mouse, previous data indicated two distinct microglia functional states of 'desensitization'

114 induced by an intraperitoneal LPS challenge (Schaafsma et al., 2015) and 'priming' during 115 accelerated aging resulting from deficiency of the DNA-damage repair protein Ercc1 (D. D. A. 116 Raj et al., 2014). These different functional states can be unmasked by a (secondary) LPS 117 stimulus resulting in a 'tolerant' or 'trained' immune response, respectively, and were so far characterized based on the analysis of limited sets of genes by qPCR (D. D. A. Raj et al., 2014;

119 Schaafsma et al., 2015). For several tested inflammatory genes, such as $/ / 1 b, T n f$, and $/ / 6$, the 120 initial stimulus determined whether microglia show a dampened or enhanced response to 121 (secondary) LPS treatment. However, the genome-wide transcriptional remodeling in 122 desensitized and primed microglia and its effect on responsiveness to future inflammatory 123 exposure are unknown. Therefore, we performed RNA-sequencing on acutely isolated, FACS124 purified microglia from mice that were either recurrently treated with LPS with a 1-month 125 interval, or from Ercc1 ${ }^{\Delta / k o}$ mice that were stimulated with LPS near the end of their lifespan at 10-12 weeks of age (Figures 1, S1, S2A, S2B, Supplemental file 1, 2).

For the tolerance model, we analyzed four treatment groups: the controls that were treated with PBS twice (PP), mice that were treated with LPS and after 1 month with PBS (LP) to investigate desensitization, mice treated with PBS followed by LPS after 1 month to determine the acute response to LPS (PL) and mice that were treated with LPS twice with a 1-month interval between challenges (LL) to identify the tolerant response (Figure 1). I.p. injection of LPS resulted in significant changes in gene expression in microglia after 3 hours (Figures 1B, 1D, S2A). After 1 month, this initial response to LPS had subsided and in terms of the transcriptional program, no significant differences were observed between the PP and LP groups (Figures 1D, S2A). However, when mice were challenged with LPS for a second time, the response was different from the initial response (Figure S2A) and many genes were significantly differentially expressed between PL and LL conditions (Figure 1B, 1D).

For the microglia priming model, both the Ercc1 ${ }^{\Delta / k o}$ mice and their control littermates were treated with PBS (WT-PBS, KO-PBS) to identify priming effects or with LPS (WT-LPS, KOLPS) to identify training. As we observed previously, deletion of Ercc1 in itself results in significant changes in gene expression (Figures 1E, S2B). However, when Ercc1 ${ }^{1 / k o}$ mice were treated with LPS, the difference between microglia from control and knockout mice was much more pronounced (Figures 1C, 1E, S2B).

The response to an acute LPS stimulus was highly similar in mice of the tolerance (C57BL/6J) and priming (FVB/ C57BL/6J) model. Nevertheless, it cannot be fully excluded that 
146 the LPS response is slightly affected by the genetic backgrounds of the two mouse strains

147 used in this study.

148

149 Many genes differentially expressed between PP versus LP and WT-PBS versus WT-LPS

150 showed very similar changes in expression in response to LPS, after ranking them based on

151 expression level and comparing rank positions between the two groups (Figure S2C). With our

152 RNA-sequencing dataset, we confirmed several of our previous findings (Holtman et al., 2015;

153 D. D. A. Raj et al., 2014), and replenish this information with complete gene expression profiles

154 of the desensitized and primed microglia phenotype. The opposite regulation of the pro-

155 inflammatory genes $/ / 1 \mathrm{~b}$ in tolerant (LL) and trained (KO-LPS) microglia (Figure S2D, S2E)

156 was confirmed. In addition, primed microglia (KO-PBS) showed increased expression of genes

157 belonging to the 'primed' gene hub (Holtman et al., 2015), including Clec7a and Axl when

158 compared to control animals (WT-PBS, Figure S2E). 


\section{Genes with distinct transcriptional responses to LPS have different biological} functions

162 Following 3 hours of LPS exposure (PL versus PP, LogFC>1, FDR <0.01), 1489 genes showed 163 increased expression (Figures 1D, 2A) while 1474 genes were downregulated in microglia 164 (Figures 1D, S3A). Generally, LPS-induced genes were involved in various aspects of the immune response (Figure S3B, Supplemental file 3), while genes downregulated by LPS were involved in multiple biological processes (Figure S3C, Supplemental file 3). Of note, in the LPS-downregulated genes, the association with biological processes showed a lower level of significance than the GO terms associated with LPS-upregulated genes (Figures S3B, S3C, Supplemental file 3).

Focusing on LPS-induced genes, out of 1489 genes, 1187 responded similarly in case of re-stimulation with LPS (cluster 1 and 3), while 302 showed a reduced response to a second LPS challenge (cluster 2 and 4, Figure 2A). Processes uniquely associated with the 1187 responsive genes were 'ribosome biogenesis', 'regulation of cytokine production', and 'inflammatory response', while 'positive regulation of immune response' and 'response to bacterium' were particularly associated with the 302 tolerized genes (Figure 2C, Supplemental file 3). This is in line with the finding that tolerant monocytes/macrophages are impaired in their ability to produce pro-inflammatory cytokines (Foster et al., 2007; Novakovic et al., 2016), but are capable of expressing genes involved in damaging or killing pathogens, so-called antimicrobial effectors. These data suggest that an i.p. injection with LPS initially induces a major immune response in microglia, which then results in the establishment of long-term innate immune tolerance that is characterized by a significantly reduced transcriptional response to secondary LPS treatment.

\section{Primed microglia have a genome-wide exaggerated response to LPS treatment}

To gain insight into the biological processes affected by Ercc1 deletion in microglia from unstimulated and LPS-treated mice, Manhattan distance-based hierarchical clustering analysis of genes followed by gene ontology analysis per cluster was performed (Figures 2B, 2D, Supplemental file 4). Seven clusters were identified containing genes that were altered by Ercc1 deletion (KO). Genes of clusters 5 are similarly affected in WT and KO microglia and downregulated in both genotypes after LPS treatment. GO terms associated with these genes included 'regulation of cell cycle phase transition', and 'pattern specification process'. The expression of genes in cluster 7 are induced in KO compared to WT microglia and are depleted

193 in both conditions after LPS treatment. These genes are involved in processes like 'nuclear 194 division', 'cell division' and 'the immune response'. Cluster 6 contain genes that are upregulated in microglia of PBS and LPS-treated WT compared to KO mice. These genes are associated with 'cell junction organization' and 'cell-cell adhesion'. 
197 Cluster 3 contains genes that were induced in PBS-treated and to a greater extent in LPS-

198 treated KO compared to WT microglia. These primed genes are associated with GO terms

199 'regulation of defense response', 'cell division', 'response to bacterium' and 'innate immune

200 response'. Cluster 2 contains genes that were induced by LPS in KO and to a lesser extent in

201 WT microglia and these genes were associated with GO terms such as 'response to

202 bacterium', 'innate immune response' and 'regulation of cytokine production', underlining the

203 trained immune response of KO microglia to LPS challenge. Cluster 4 contains genes that

204 were induced by LPS in WT and to a lesser extent in KO microglia and these genes were

205 associated with GO terms such as 'trans-synaptic signaling, 'chemical synaptic transmission'

206 and 'nervous system process'. Finally, genes in cluster 1 are induced by LPS to a similar

207 degree in WT and KO microglia and are associated with GO terms, like 'signal release',

208 'regulation of cytokine production' and 'inflammatory response' (Figure 2D).

209 In agreement with our previous findings (D. D. A. Raj et al., 2014), also at a genome-wide level,

210 Ercc1 deficiency generates an environment where microglia are more responsive to

211 inflammatory stimuli, as evidenced by a large set of inflammatory genes whose expression is

212 significantly increased in microglia upon LPS treatment of Ercc1 ${ }^{\Delta / k o}$ mice. 


\section{Epigenetic remodeling in response to LPS desensitization and accelerated aging}

215 The transcriptomes of microglia from PP and LP treated mice are almost identical, however,

216 they respond very differently to re-stimulation with LPS (Figures 2A, S2A). Similarly, many 217 genes that are not transcriptionally altered in Ercc1 deficient mice show an increased 218 transcriptional response to LPS (Figures 2B, S2B). These data suggest that microglia have 219 innate immune memory that is not secured in their transcriptome. Rather, similar to macrophages and as suggested by our previous analysis of the $I 1 \beta$ locus (Schaafsma et al., 2015), it is likely that epigenetic reprogramming is involved.

To gain insight in the genome-wide epigenetic changes induced by LPS desensitization and Ercc1 deficiency, we performed assay for transposase accessible chromatin-sequencing (ATAC-seq), which indiscriminately identifies open chromatin regions in the genome (Buenrostro et al., 2013, 2015), and chromatin immunoprecipitation-sequencing (ChIP-seq), which probes histones carrying specific posttranslational modifications (Henikoff \& Shilatifard, 2011; Kouzarides, 2007). In case of the tolerance model, we used antibodies targeting $\mathrm{H} 3 \mathrm{~K} 4 \mathrm{me} 3$ and H3K27Ac to identify transcription start sites (TSSs) and enhancers of actively transcribed genes, respectively. In Ercc ${ }^{\Delta / \mathrm{ko}}$ mice, we also analyzed H3K4me3 and H3K27ac, and additionally H3K4me1 which together with $\mathrm{H} 3 \mathrm{~K} 27 \mathrm{ac}$ marks active enhancers and the Polycomb-regulated H3K27me3 associated with transcriptional repression (Figure S4A).

Representative examples of chromatin accessibility and occupation, and RNA expression of individual tolerized (I/1b, Tnf, Cc/3, Nfkb1 and Relb, Figure S2D, S4B) and primed/trained (II1b, Ccl3, Cxcl11, Clec7a and Axl, Figure S2E, S4C) genes are depicted and indicate dynamic regulation of epigenetic signatures associated with changes in gene expression levels.

\section{Epigenetic characterization of tolerized genes}

239 In order to determine which chromatin characteristics correspond to the transcriptional 240 changes induced by LPS, we identified regions in the genome with significant differences in 241 chromatin accessibility or histone modifications. Differential peaks were classified as 242 promoters when they were located within $1000 \mathrm{bp}$ downstream and $1000 \mathrm{bp}$ upstream of a 243 TSS of the nearest gene and as enhancers when being located distal of this region. To 244 integrate RNA-, ATAC-, and ChIP-seq data, the differentially expressed genes (logFC) were correlated to differentially regulated chromatin regions (M-value) within one comparison.

Similar to what has been described in macrophages (Escoubet-Lozach et al., 2011; Hargreaves et al., 2009; Saeed et al., 2014), in microglia H3K4me3 already marks TLR4responsive promoters prior to LPS stimulation (Figure S4B). Irrespective whether microglia are exposed to LPS for the first or the second time, genes which are expressed in response to 
induced gene expression significantly correlates with ATAC, H3K4me3, H3K27Ac peak enrichment, associated with permissive gene expression, in promotors and enhancers of microglia from PL compared to PP (Figure 3A left panel, Supplemental file 5) and LL compared to LP-treated mice (Figure 3A middle panel, Supplemental file 5). In line with the fact that H3K4me3 is generally associated with promoters, LPS-induced gene expression only significantly correlates with enrichment of this mark in promoters but not enhancers in the PL versus PP comparison (Figure 3A left panel, Supplemental file 5).

Tolerized genes are characterized by increased expression after the primary LPS challenge $(P L)$ and reduced induction after the secondary LPS challenge (LL) (Figure 2A). When comparing the microglia response to primary and secondary LPS challenge (PL versus $L L$ ), the expression of the tolerant genes after primary LPS challenge significantly correlated with enrichment of ATAC and H3K27ac peaks at enhancers, but not promoters (Figure 3A right panel, Supplemental file 5). This means vice versa that after secondary LPS challenge, tolerized genes were depleted in these activating expression-associated enhancer marks. The expression of tolerized genes was not significantly correlated to the promoter-associated histone mark H3K4me3. Together, these results indicate that the tolerized response of microglia to LPS seems to be mainly enhancer driven and, at least partially, explained by a loss of histone marks associated with active expression after secondary LPS challenge (Figure 3 right panel, Supplemental file 5).

Transcription factors (TFs) are critical determinants of changes in both transcriptional and epigenetic programs that can be activated by signaling pathways. TFs are often part of large, multimeric protein complexes that also contain chromatin-modifying enzymes, and recruitment of TFs can result in local remodeling of the chromatin (Zhou et al., 2017). DiffTF was used to identify the TFs that might be involved in the differential chromatin regulation in tolerant microglia. Differential chromatin accessibility peaks (weighted mean difference) of putative TF binding sites (TFBS) between two conditions were identified. Next, this ATAC-seq data was integrated with RNA-seq data by correlating differential accessible peaks of putative TFBS to differential gene expression levels of a particular TF. This procedure is then repeated for each TF. Based on whether the correlation of TF activity and expression is positive or negative, TFs were classified as an activator or a repressor. Alternatively, when there was no correlation detected, the TF was classified as undetermined or the TF was not expressed (Berest et al., 2019, Figure 3B, 3C, 3D).

Genome-wide accessible chromatin regions, significantly enriched in naïve microglia (PP, Figure 3B, Supplemental file 6), contain binding sites for the key myeloid TFs PU.1 (SPI1), IRF8 and MAFB, described to be crucial for adult mouse microglia transcriptional identity (Kierdorf et al., 2013; Matcovitch-Natan et al., 2016). In addition, naïve microglia are enriched 
is critical for the microglia homeostatic signature (Butovsky et al., 2014). Binding sites of homeostasis-associated TFs were lost and TFBS of known mediators of LPS-induced inflammatory pathways in macrophages/microglia (Escoubet-Lozach et al., 2011; Holtman et al., 2015; Roach et al., 2007) including the NF-kB TF family (NFKB1/2, REL/RELB, Oeckinghaus \& Ghosh, 2009), TFs involved in the immediate early response (IER; JUN, JUNB, FOSL2) and the interferon pathway (IRF TF family), STAT3, CEBPB (Simpson-Abelson et al., 2017; Straccia et al., 2011; Valente et al., 2013), and the general activating transcription factor ATF1 were all detected to be enriched in microglia acutely challenged with LPS (PL versus PP, Figure 3B, Supplemental file 6).

After a secondary LPS challenge, in tolerant microglia (LL vs. LP), many of these inflammatory-associated TFBS are still enriched, except those belonging to the NF-KB TF family, indicating that recruitment of these TFs specifically occurs after primary LPS challenge. This is also confirmed in the direct comparison of acutely stimulated versus tolerant microglia (PL vs. LL, Figure 3D, Supplemental file 6). Furthermore, the enrichment of TFBS for SPI1, IRF8, CTCF and MAF, important for the homeostatic microglia transcriptome (Gosselin et al., 2014; Matcovitch-Natan et al., 2016), in desensitized microglia (LP vs. LL, Figure 3C, Supplemental file 6) explains their naive-like transcriptome (Figure 1D).

Many of the inflammatory-associated putative TFBS are depleted and the TFBS for the transcriptional repressor ZEB1, associated with suppression of immune active genes (C. J. Block et al., 2019; Wang et al., 2009), are enriched in tolerant microglia (LL) when compared to microglia of acutely LPS-challenged mice (PL, Figure 3D, Supplemental file 6), possibly explaining the dampened expression of tolerized genes in LL microglia.

These data indicate that deposition of permissive chromatin marks drive the acute LPSresponse of microglia, and loss of those, in particular surrounding TFBS of the NF-KB family, might at least partially explain the tolerized response of microglia to a secondary LPSchallenge. 


\section{Epigenetic characterization of the priming response}

In case of microglia priming, we also observed a general concordance between the transcriptional changes following Ercc1 deficiency and LPS challenge and the presence of permissive chromatin characteristics. Induction of gene expression by Ercc1 KO or by LPS in both WT and KO microglia significantly correlated with increased chromatin accessibility in promoters as well as enhancers (Figure 4A, Supplemental file 2 and 7 ). In addition, compared to WT-PBS, many KO-induced genes are marked with significant enrichment of the permissive marks H3K27Ac and H3K4me3 (Figure 4B). The expression of some of the KO-induced genes additionally correlates with $\mathrm{H} 3 \mathrm{~K} 4 \mathrm{me} 1$ enrichment, which together with $\mathrm{H} 3 \mathrm{~K} 27 \mathrm{ac}$ deposition is associated with active transcription (Creyghton et al., 2010). Inversely, some of the genes whose expression is induced by Ercc1 deficiency are depleted in H3K27me3, which is associated with Polycomb-associated gene repression, at promoters of microglia from KO versus WT mice (Figure 4B). Together, this indicates that the expression of primed genes in Ercc1 deficient mice is driven by enriched chromatin characteristics associated with permissive transcription and depletion of repressive chromatin marks.

We next determined accessible conserved TFBS and corresponding expression of the TFs in microglia of (LPS-treated) Ercc1 deficient and WT mice. Compared to controls, SMAD1/3/4 binding sites are lost in microglia of $\mathrm{KO}$ mice (Figure $4 \mathrm{C}$, Supplemental file 8), which are involved in maintenance of the microglia homeostatic gene signature (Butovsky et al., 2014; Massagué et al., 2005). Generally, immune activation of microglia results in the loss of the homeostatic signature (Holtman et al., 2015; Keren-Shaul et al., 2017; Krasemann et al., 2017; Zrzavy et al., 2017), and our data show that this is also true in primed microglia (Figure 4E). Microglial TF motifs with increased chromatin accessibility upon Ercc1 deletion include TFs whose associated functions were previously attributed to primed microglia (Holtman et al., 2015; D. D. A. Raj et al., 2014), namely lysosomal biogenesis (TFE3, Brady et al., 2018), inflammation (CEBP TF family (Simpson-Abelson et al., 2017; Straccia et al., 2011; Valente et al., 2013), IER TF family, ATF1) and proliferation (CEBP TF family, Gómez-Nicola et al., 2013; Pal et al., 2009) (Figure 4C, Supplemental file 8).

In contrast to microglia of LPS-treated WT mice, trained microglia of LPS-treated KO mice are enriched in accessible TF motifs for regulators with known roles in acute LPS-induced inflammation (Escoubet-Lozach et al., 2011; Holtman et al., 2015; Roach et al., 2007), including NFKB2 and REL/RELB, several members of the IRF TF family (IRF7, 8, 9), and IERrelated TFs. In addition, ZEB1, associated with immune response suppression (C. J. Block et al., 2019; Wang et al., 2009), is depleted in trained microglia (Figure 4D, Supplemental file 8). Together with the fact that homeostatic genes in microglia of LPS-treated KO mice are even further downregulated than in $\mathrm{KO}$ microglia (Figure 4E), these results underline the training of microglia from KO mice. 
bioRxiv preprint doi: https://doi.org/10.1101/2021.05.30.446351; this version posted May 31, 2021. The copyright holder for this preprint

(which was not certified by peer review) is the author/funder, who has granted bioRxiv a license to display the preprint in perpetuity. It is made available under aCC-BY 4.0 International license.

354 These data suggest that Ercc1 depletion shapes a chromatin landscape that enables both

355 the loss of the microglia homeostatic signature, and the gain of a transcriptional profile

356 associated with inflammation, which is enhanced with LPS challenge. 


\section{A large proportion of tolerized genes show an increased transcriptional response in} primed and trained microglia

Both in tolerized (cluster 2 and 4, Figure 2A) and primed/trained gene sets (cluster 1, 2 and 4, Figure 2B), immune system processes were significantly enriched (Figure 2C, 2D). We intersected these gene sets and not only were similar biological processes affected, but many of the differentially regulated genes were also shared. Out of the 302 tolerized genes, 145 genes overlap with acute LPS response-induced genes and 46 showed a significantly higher expression level in microglia of LPS-treated mice and Ercc1 ${ }^{\Delta / k o}$ mice after LPS treatment. 264 and 249 genes were uniquely enriched in primed (KOPBS versus WT-PBS) and trained (KO-LPS versus WT-LPS) microglia, respectively, and 251 genes overlapped between these conditions. Finally, 103 overlapping genes were enriched in acutely challenged, tolerized, primed as well as trained microglia (Figure 5A, Supplemental file 9). Significantly associated biological processes within these gene sets were identified (Figure 5B, Supplemental file 10). Genes involved in 'organelle fission', 'nuclear division' and 'chromosome segregation' were associated with and limited to primed microglia from Ercc1 deficient mice. The genes exclusive for training are involved in 'response to oxidative stress' and 'ribosomal small subunit assembly'. The 251 genes that are shared between primed and trained microglia are associated with 'positive regulation of cytokine production' and 'regulation of immune effector process'. The acute, tolerized and trained gene sets, with or without the primed gene set, share GO terms such as 'regulation of innate immune response', 'NF-kappaB signaling' and 'regulation of apoptotic signaling pathway'.

In order to determine possible regulators of the opposing LPS response between tolerized and trained genes, motifs for TFBS in genomic regions with enriched chromatin accessibility were identified with diffTF in acute (PL versus PP), tolerant (LL versus $P L)$, primed (KO-PBS versus WT-PBS) and trained (KO-LPS versus WT-LPS) microglia (Figure 5C, Supplemental file 6,8 ). The four identified microglial phenotypes (acute, tolerized, primed, trained) seem to 383 be regulated by specific TF networks, explaining why gene sets, although being partially shared between some or all of the four phenotypes, are regulated in opposite directions.

Summarizing, the presented data indicate that microglia in vivo possess innate immune memory and that different types of stimuli, in this case Ercc1 deficiency or LPS, can leave epigenetic imprints which seem to influence the response towards a secondary challenge leading to microglia training or tolerance to LPS. Condition-specific epigenetic profiles seem to involve the activity of specific TF networks, which might drive the opposite regulation of shared genes in trained and tolerant microglia. 


\section{Discussion}

392

393

394

395

396

397

398

399

400

401

402

403

404

405

406

407

408

409

410

411

412

413

414

415

416

417

418

419

420

421

422

423

424

425

426

427

Monocytes and tissue resident macrophages play important roles in development, metabolism and immunity, thereby contributing to the maintenance of homeostasis. Though they are innate immune cells, macrophages can retain information of past inflammatory events, resulting in an altered response to reinfection. Depending on the primary trigger, macrophages can become 'tolerant', showing hypo-responsiveness, or 'trained' with increased responsiveness to subsequent stimuli. Biologically, these mechanisms are generally thought to provide a survival advantage in case of trained immunity (Netea, 2013), while the refractory state of tolerant macrophages causes increased mortality (Biswas \& Lopez-Collazo, 2009). However, these effects seem to be context-dependent and it was hypothesized that trained immunity might have deleterious consequences in autoimmune diseases (Arts, Joosten, et al., 2018), whereas tolerance can provide a protective mechanism limiting the toxic effects of prolonged inflammation (Seeley \& Ghosh, 2017).

Monocytes/macrophages undergo functional programming after exposure to microbial components (Kleinnijenhuis et al., 2012; Novakovic et al., 2016) and the associated genomewide epigenetic characteristics of innate immune memory have been described over the past years (Arts, Moorlag, et al., 2018; Glass \& Natoli, 2016; Novakovic et al., 2016; Perkins et al., 2016; Saeed et al., 2014). These observations are thought to provide clues as to which pathways to target to reverse 'tolerance' or stimulate 'training' in a clinical setting.

The CNS parenchyma contains microglia, tissue resident macrophages that fulfill highly specialized functions extending far beyond their innate immunological functions (Eggen et al., 2013; Eggen et al., 2017). Besides their different job-description that is attuned to their CNS environment, in contrast to some other tissue-derived macrophages, microglia also have a relatively long lifespan (Askew et al., 2017; Eggen et al., 2017; Füger et al., 2017; Tay et al., 2017). Microglia longevity together with the long-lasting nature of epigenetic mechanisms can have drastic effects on brain functioning and cognition.

In microglia, altered functional outcomes reminiscent of 'tolerance' and 'training' have been described and these mechanisms might contribute to poor cognitive outcomes in sepsis patients (Semmler et al., 2013), the general aged population and neurodegeneration (Haley et al., 2017; Knuesel et al., 2014; Neher \& Cunningham, 2019; Pardon, 2015; Perry \& Holmes, 2014; Wendeln et al., 2018). Particularly, disease features in mouse AD and stroke models appear to be altered in animals where microglia were exposed to systemic inflammatory stimuli (Wendeln et al., 2018).

Here, we show that exposure of microglia to an inflammatory challenge (LPS) or an environment of accelerated aging in vivo results in substantial transcriptional and epigenetic changes that impact on their future ability to mount an inflammatory response. In particular, we found that approximately 103 genes are oppositely regulated when 'desensitized' or 
'primed' microglia are exposed to i.p. injection of LPS and that these genes are involved in inflammatory and apoptotic processes.

In the control situation, promoter and cis-regulatory elements controlling these inflammatory genes are characterized by a certain degree of chromatin accessibility, as well as $\mathrm{H} 3 \mathrm{~K} 4 \mathrm{me} 3$ and H3K27Ac enrichment. In agreement with increased transcription of inflammatory genes in microglia from mice treated with LPS, these chromatin parameters were increased during the acute response. In case of tolerance, abundance of these marks is decreased after LPS reexposure, which, at least partially, explains compromised induction of gene expression after secondary LPS challenge. Possibly, there is a second layer of gene expression repression by inhibitory histone marks. Previous data suggests a role for the inhibitory histone marks H3K9me2/3 in this context (Perkins et al., 2016; Schaafsma et al., 2015). The TF RELB has a recruiting role for $\mathrm{H} 3 \mathrm{~K} 9 \mathrm{me} 2 / 3$ at the $/ 1 \beta$ locus after LPS challenge, which leads to transcriptional repression of $/ 11 \beta$ in response to a secondary LPS challenge (El Gazzar et al., 2008; Schaafsma et al., 2015). We identified enriched accessible binding motifs for REL and RELB in PL versus LL microglia genome-wide, indicating that a primary LPS challenge might lead to recruitment of REL/RELB at regulatory elements of tolerized genes and might inhibit gene expression upon secondary LPS challenge through recruitment of H3K9me2/3. However, this hypothesis needs to be confirmed in future ChIP-sequencing experiments.

In case of priming, continuous exposure to an aging environment results in increased chromatin accessibility as well as $\mathrm{H} 3 \mathrm{~K} 4 \mathrm{me} 3$ and $\mathrm{H} 3 \mathrm{~K} 27 \mathrm{Ac}$ enrichment. These changes are associated with increased gene expression levels in Ercc1 ${ }^{\Delta / k o}$ microglia as well as after an LPS exposure. SMAD binding elements are known to act collaboratively with PU.1 and other TFs to facilitate transcription of the homeostatic microglia signature (Holtman et al., 2017). In the accelerated aging model, chromatin signatures associated with active gene expression are less associated with SMAD binding elements. This is accompanied by a decrease in expression of homeostatic microglia signature genes in Ercc1 $1^{\Delta / k o}$ microglia, especially following LPS treatment. Microglia priming in this model is caused by neuronal genotoxic stress, since only Ercc1 deficiency in neurons, but not astrocytes and microglia induced microglia priming (D. D. A. Raj et al., 2014; Zhang et al., 2020). While active marks on promoters and enhancers correlate with increased expression, the Polycomb regulated repressive mark H3K27me3 is lost in some genes whose expression is increased in Ercc1 $1^{\Delta / k o}$ microglia. Loss of the Polycomb mark H3K27me3 could be a critical determinant of cellular identity and function of primed microglia as the Polycomb repressive complex 2 (PRC2) is involved in maintenance of homeostatic microglia identity in different CNS brain regions. Loss of PRC2 activity in microglia resulted in aberrant gene expression and altered functionality (Ayata et al., 2018). 
exacerbation of $\beta$-amyloidosis (Wendeln et al., 2018). Although the hyperresponsive nature of

466 microglia to two stimuli seems to be comparable in these two studies, the underlying molecular

467 mechanisms might be different due to the fact that the LPS stimulus and AD pathology were

468 separated by a non-inflammatory phase (Wendeln et al., 2018), while persistent microglial

469 activation is present in $\operatorname{Ercc} 1^{\Delta / k o}$ mice.

470 Though the genes involved in tolerance and training are overlapping, the fact that the

471 chromatin composition in these regions is diverse, suggests the involvement of distinct protein

472 complexes and epigenetic enzymes. Summarizing, different molecular pathways and different

473 epigenetic mechanisms regulate the behavior of inflammatory genes in 'tolerant' or 'trained'

474 microglia.

475 Our data provides evidence that at least one type of macrophage, the CNS endogenous

476 microglia, in vivo can adopt epigenetic programs that contribute to the establishment of

477 different functional phenotypes and thereby influence neuroinflammation in the long-term. 
A
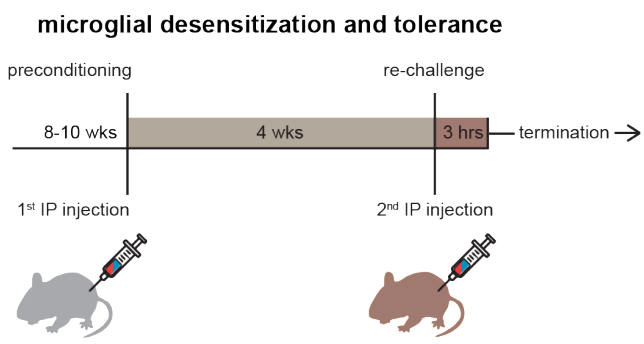

brain isolation

microglial priming and training
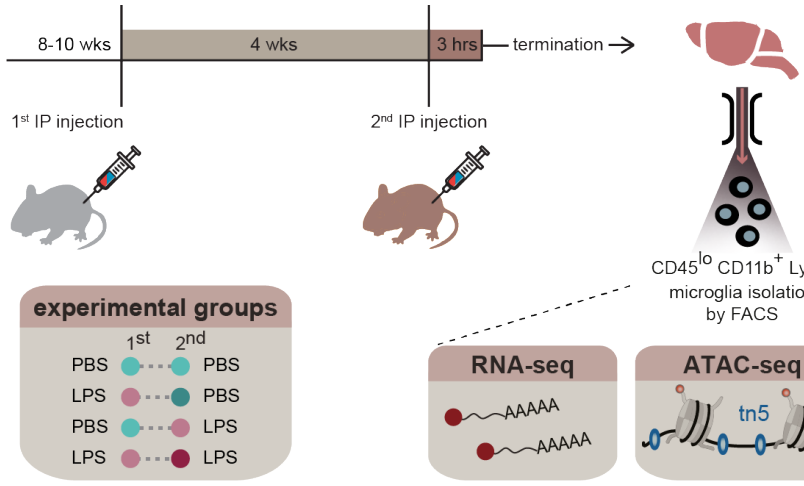

$\mathrm{CD} 45^{\mathrm{lo}} \mathrm{CD} 11 \mathrm{~b}^{+} \mathrm{Ly}_{6 \mathrm{c}}$

microglia isolation
by FACS

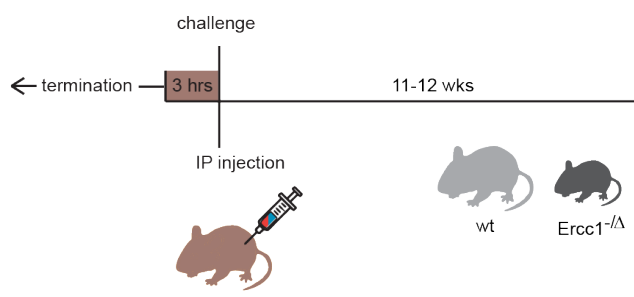

B

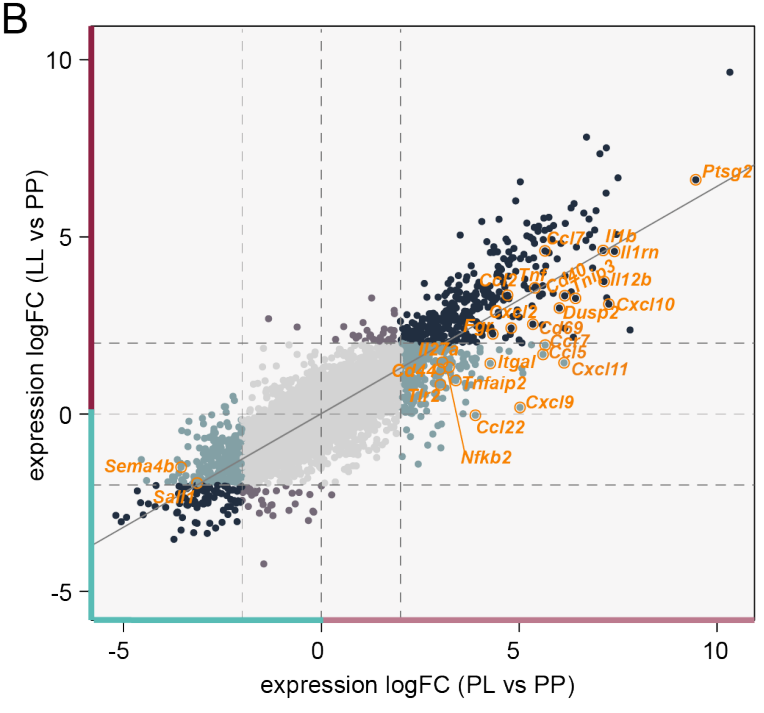

C
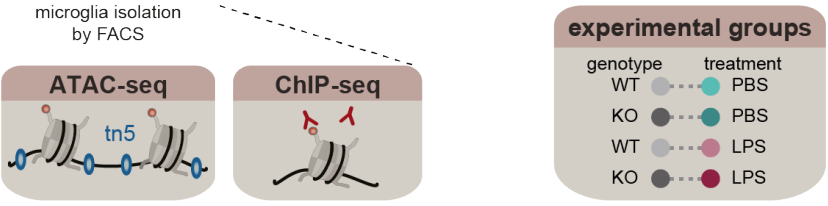

D
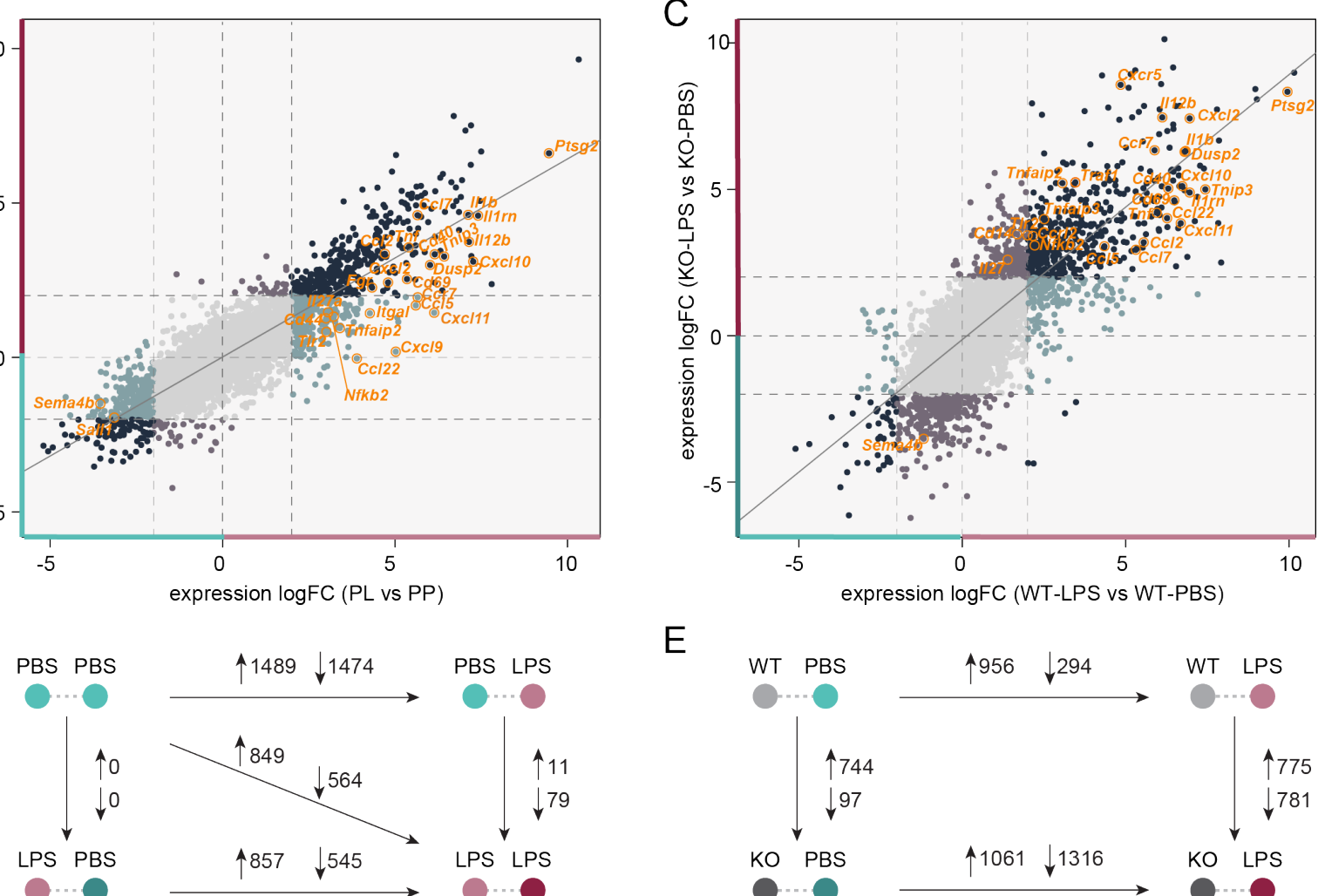

E

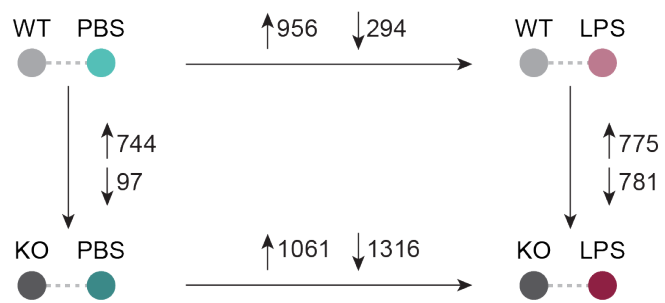

Figure 1, LPS desensitization and accelerated aging result in distinct changes of the microglia immune response.

a, Graphic representation of the mouse models and treatment groups. A pure microglia population was isolated by FACS and subjected to RNA, ATAC, and ChIP-sequencing analysis. b, c, Four-way plots depicting changes in gene expression in microglia isolated from LPS-injected naive and pre-conditioned mice $(n=3$ per experimental condition) (b) and Ercc $1^{\Delta / k o}$ and control mice ( $n=3$ per experimental condition) (c). Every gene is represented by an individual dot. Differentially expressed genes (LogFC $>2$ ) are labeled with different colors indicating their respective expression changes. Dark blue dots indicate genes differentially expressed in both comparisons; turquoise (PL versus PP and WT-LPS versus WT-PBS) and lavender (LL versus PP and KO-LPS versus KO-PBS) dots represent genes differentially expressed in one of the comparisons, several relevant genes are highlighted. d, e, The number of differentially expressed genes ( $\mathrm{LogFC}>1$ and FDR $<0.01)$ between treatment groups in the endotoxin tolerance (d) and Ercc $1^{1 / k o}-$ induced microglia priming models (e). Upward arrows 
bioRxiv preprint doi: https://doi.org/10.1101/2021.05.30.446351; this version posted May 31, 2021. The copyright holder for this preprin (which was not certified by peer review) is the author/funder, who has granted bioRxiv a license to display the preprint in perpetuity. It is made available under aCC-BY 4.0 International license.

A

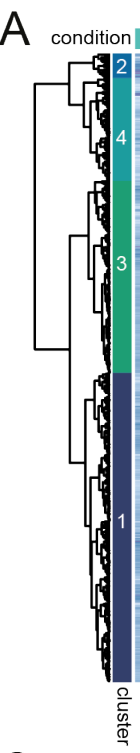

$\mathrm{C}_{\text {ate }}$

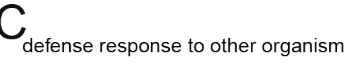

response to bacterium

blood vessel morphogenesis

blood vessel development

regulation of defense response

inflammatory response

positive regulation of immune response

activation of immune response

cell chemotaxis

regulation of ERK1 and ERK2 cascade and primed microglia.
B

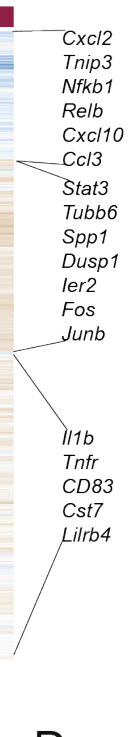

D

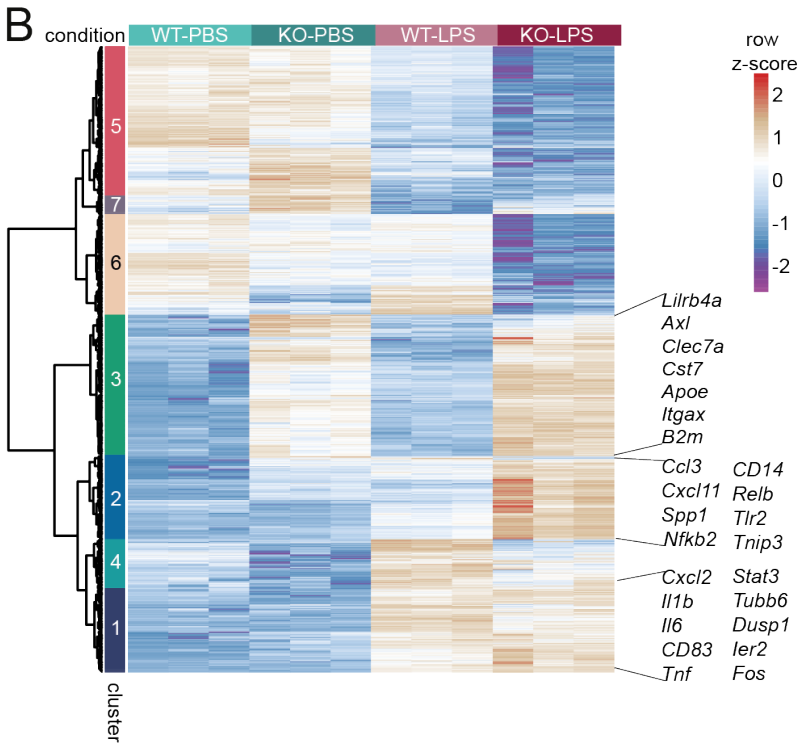

plas. mem. bounded cell projection assem. nuclear division

adaptive immune response vasculature development

cell morphogen. involved in differentiation involved in differentiation
cell junction organization cardiovascular system development
eg. of mitotic cell cycle phase transition regulation of cell cycle phase transition pattern specification process embryonic organ development cilium organization trans-synaptic signaling chemical synaptic transmission
behavior anterograde trans-synaptic signaling regulation of defense response cell division response to bacterium regulation of immune response innate immune response signal release regulation of MAPK cascade regulation of cytokine production nervous system process

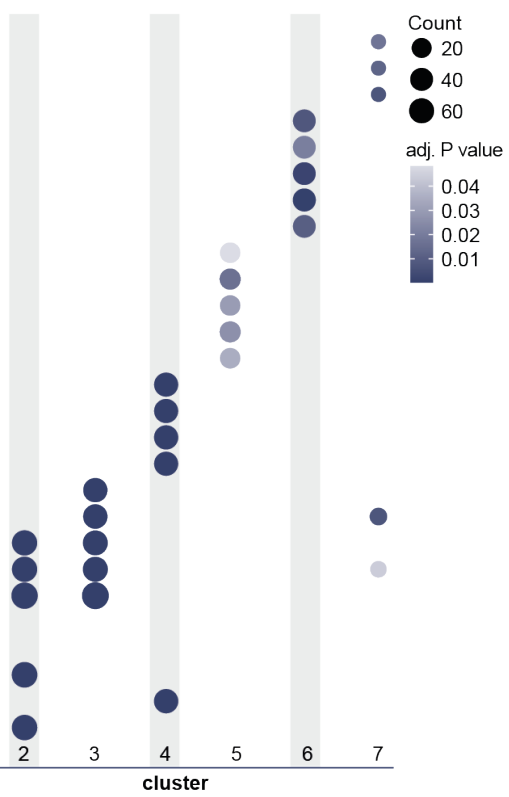

Figure 2, Identification of gene clusters with distinct transcriptional programs in desensitized

a, b, Heatmaps with Manhattan distance-based hierarchical clustering analysis of upregulated genes in response to LPS in microglia of C57/BL6 mice three hours after i.p. injection with LPS (LogFC $>1$ and FDR<0.01, PL versus PP). Four main clusters are identified, containing tolerized genes (cluster 2 and 4 ) and responsive genes (cluster 1 and 3 ) that show distinct activity to LPS re-stimulation. b, Heatmap with Manhattan distance-based hierarchical clustering analysis of all genes differentially expressed between $\operatorname{Ercc}^{\Delta / \mathrm{ko}}(\mathrm{KO})$ and Ercc $1^{\text {wt/ko, }}$, $E r c c 1^{w t /}$, Ercc $1^{\text {wt }} w^{w t}$ (WT) mice with or without LPS injection ( $\mathrm{n}=3$ per experimental condition). Seven clusters are identified, including two clusters of genes primed and trained to LPS treatment in KO mice (cluster 3 and 2). c, d, Top 5 GO annotations, based on gene count per 
GO term, of responsive (cluster 1, 3) and tolerized (cluster 2, 4) gene clusters (c) and the 7 clusters identified in $\operatorname{Ercc}^{1 / \mathrm{ko}}$ microglia (d).

A
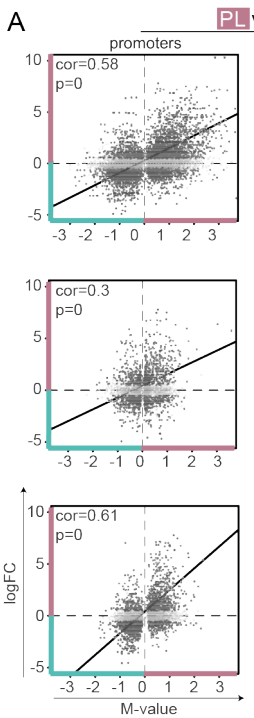

B

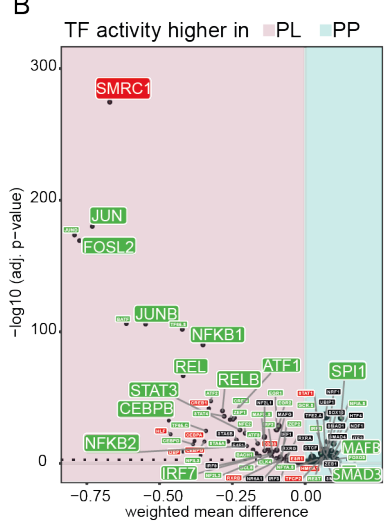

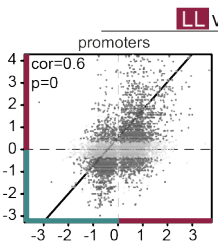
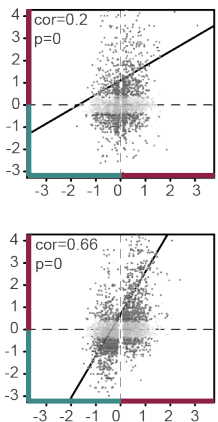

C

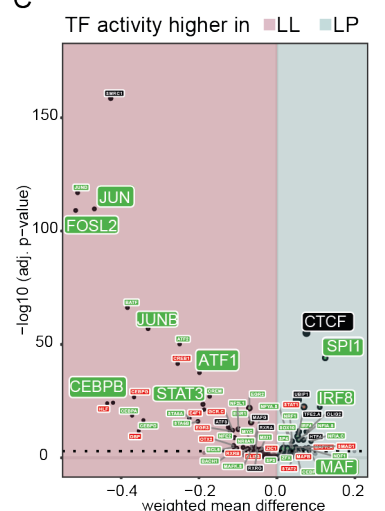

LLvsLP
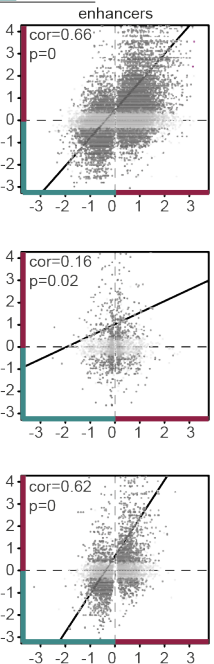

$\mathrm{D}$

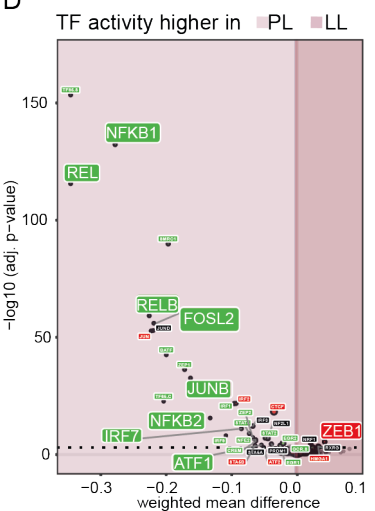

PLvsLL
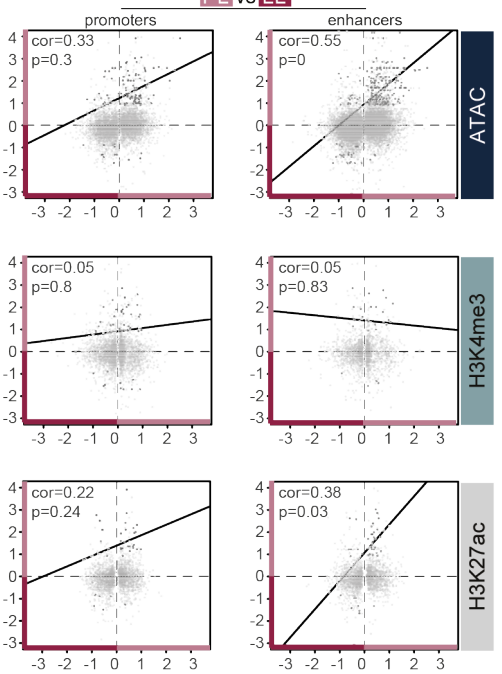

dj. $p$-value $<0.001$

no
oyes

TF class (stringency: 0.001) - undetermined - repressor

TFBS

- 20000

$\circ 30000$

Figure 3, The LPS response in naive and desensitized microglia is defined by enhancer signatures of transcriptional permissive marks.

a, Scatterplots depicting the correlation of differentially expressed genes (logFC) with corresponding differential ATAC, H3K4me3 or H3K27ac peaks (M-value) at promoters (within $2 \mathrm{~kb}$ of the nearest TSS) or enhancers (distal to promoters) between PL versus PP (left panel), LL versus LP (middle panel), PL versus LL (right panel). Each dot represents a differentially expressed gene that is associated with a significant differential chromatin peak (FDR<0.0) 1 in the given comparison. Light gray-colored dots indicate non-significant gene expression differences (FDR>0.01).

b, c, d, Transcription factor binding site analysis generated by diffTF to identify critical regulators for different gene sets based on ATAC- and RNA-seq data. Volcano plots depicting weighted mean difference of accessible TFBS between PL versus PP (b), LL versus LP (c), or $P L$ versus $L L$ (d). The color of each TF indicates its classification into an activator (green), a repressor (red) or undetermined (black) based on correlation of TFBS accessibility with RNA expression of the TF. FC=fold change, TF=transcription factor, TFBS=transcription factor binding site. 
bioRxiv preprint doi: https://doi.org/10.1101/2021.05.30.446351; this version posted May 31, 2021. The copyright holder for this preprint (which was not certified by peer review) is the author/funder, who has granted bioRxiv a license to display the preprint in perpetuity. It is made available under aCC-BY 4.0 International license.

A
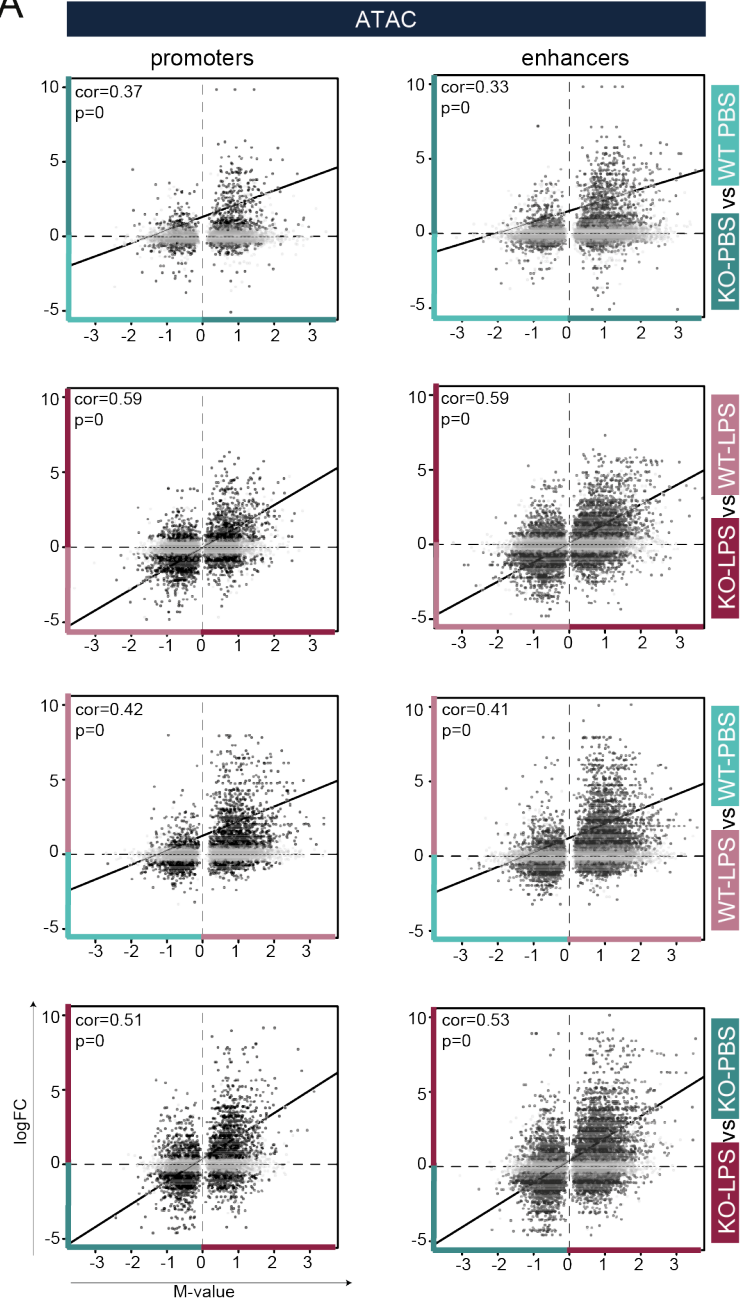

C
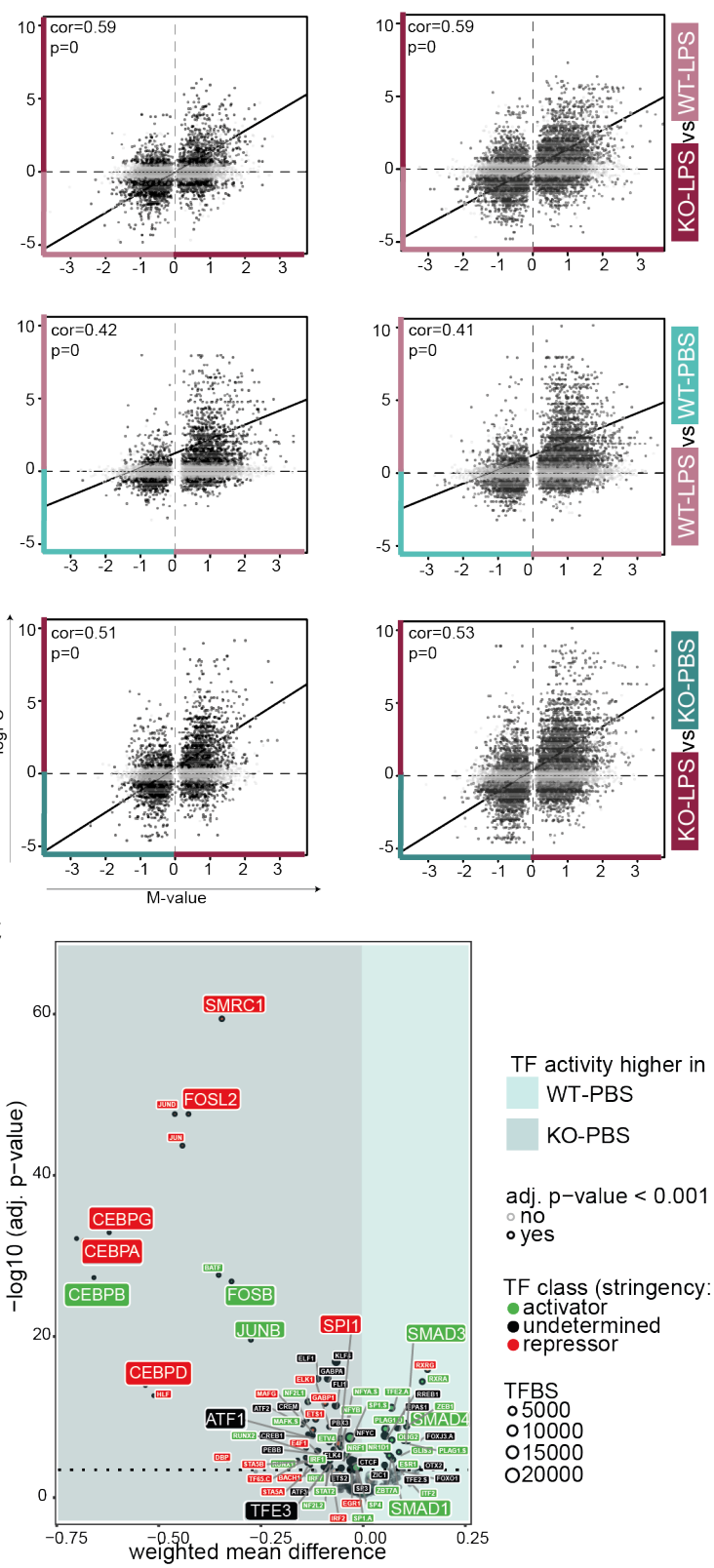

B
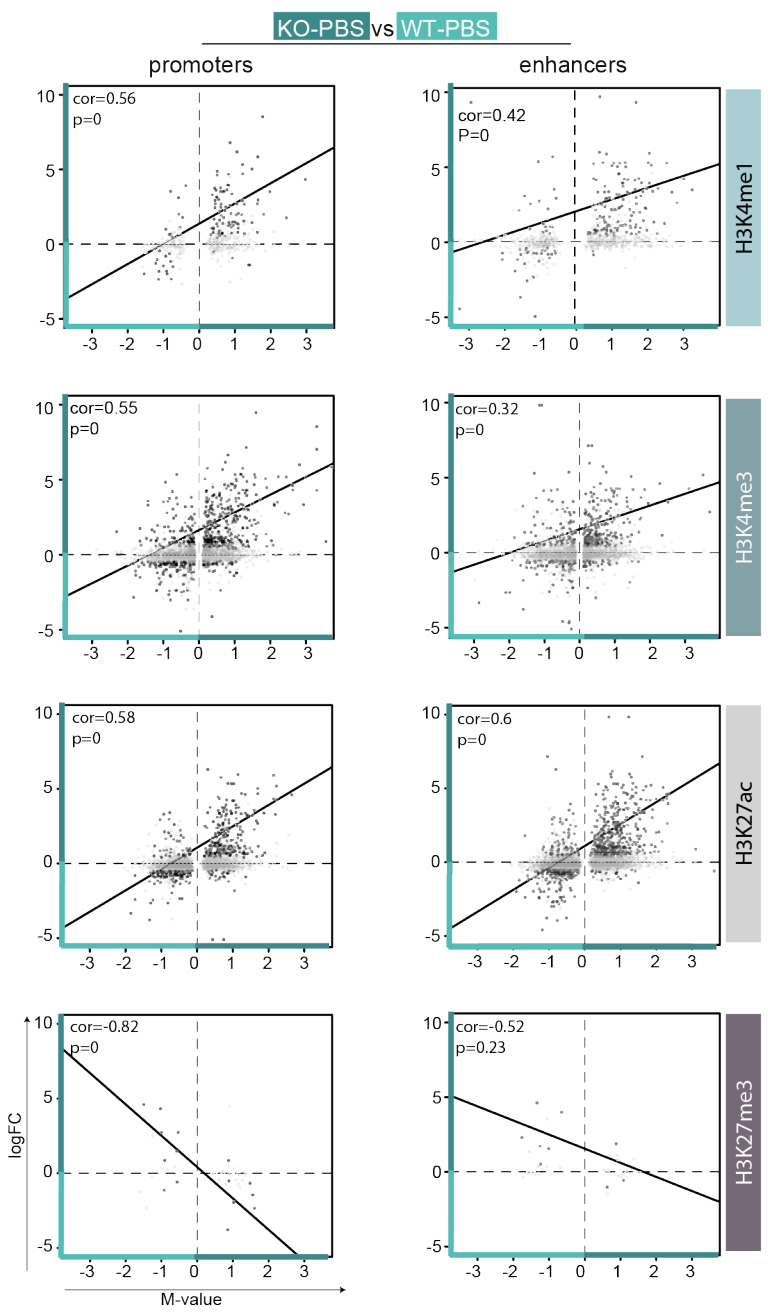

$\mathrm{D}$

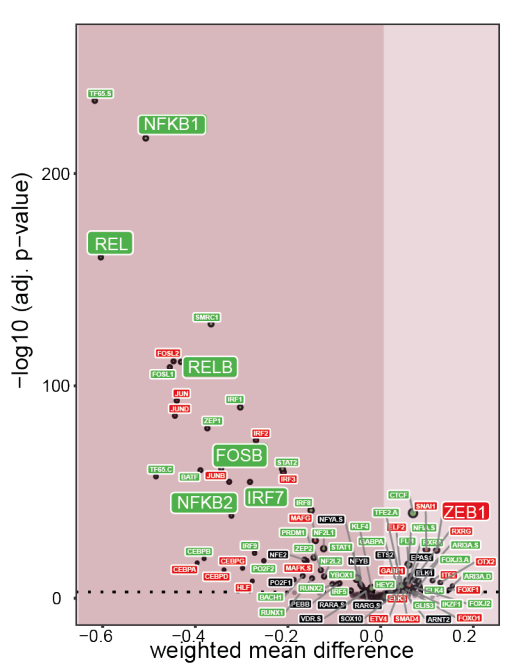

F activity higher in WT-LPS

KO-LPS
TF activity higher in
WT-PBS
KO-PBS

adj. $p$-value $<0.001$ oyes

TF class (stringency: 0.001) - activator - repressor

TFBS

$\circ 5000$
010000

015000

020000
E

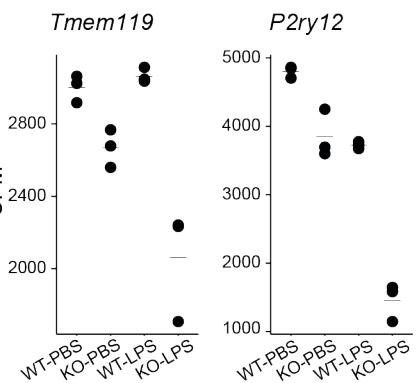

Csfir

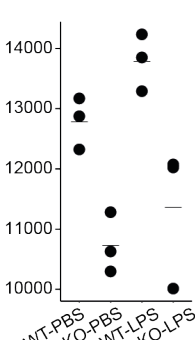

Cx3cr1

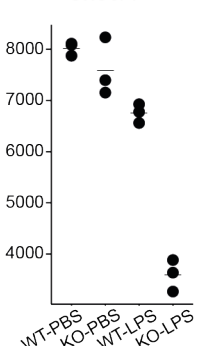

Sall1

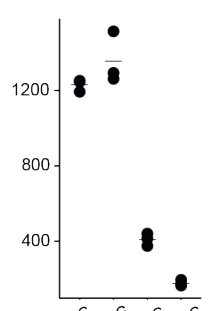

Gpr34

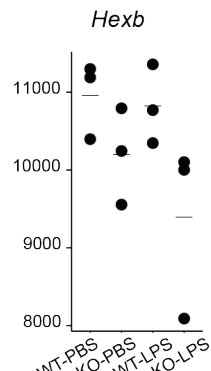


531 Figure 4, Enhancer and promoter signatures of transcriptional permissive marks regulate 532 training in primed microglia.

533 a, b, Scatterplots depicting the correlation of differentially expressed genes (logFC) with 534 corresponding differential ATAC peaks (M-value) in KO versus WT, LPS-treated KO versus 535 LPS-treated WT, LPS-treated WT versus WT and LPS-treated KO versus KO microglia (a), 536 and differential H3K4me1, H3K4me3, H3K27ac or H3K27me3 peaks (M-value) in KO versus 537 WT microglia (b). The chromatin peaks are divided into promoters (blue, within $2 \mathrm{~kb}$ of the 538 nearest TSS) and enhancers (purple, distal to promoters). Each dot represents a differentially 539 expressed gene that is associated with a significantly differential chromatin peak (FDR<0.01) 540 in the given comparison. Gray color of dots indicates non-significant gene expression 541 differences (logFC >1, FDR>0.01).

542 c, d, Transcription factor binding site analysis generated by diffTF to identify critical regulators 543 for different gene sets based on ATAC- and RNA-seq data. Volcano plots depicting weighted 544 mean difference of accessible TFBS between KO-PBS versus WT-PBS (c) and KO-LPS 545 versus WT-LPS (d) microglia. The color of each TF indicates its classification into activator 546 (green), repressor (red) or undetermined (black) based on correlation of TFBS accessibility 547 with RNA expression of the TF.

548 e, Gene expression values (CPM, Supplemental file 2) of selected homeostatic microglia 549 genes in the primed mouse model. Every dot depicts an individual animal ( $\mathrm{n}=3$ per 550 experimental condition). $\mathrm{CPM}=$ counts per million reads, $\mathrm{TF}=$ transcription factor, 551 TFBS=transcription factor binding site. 


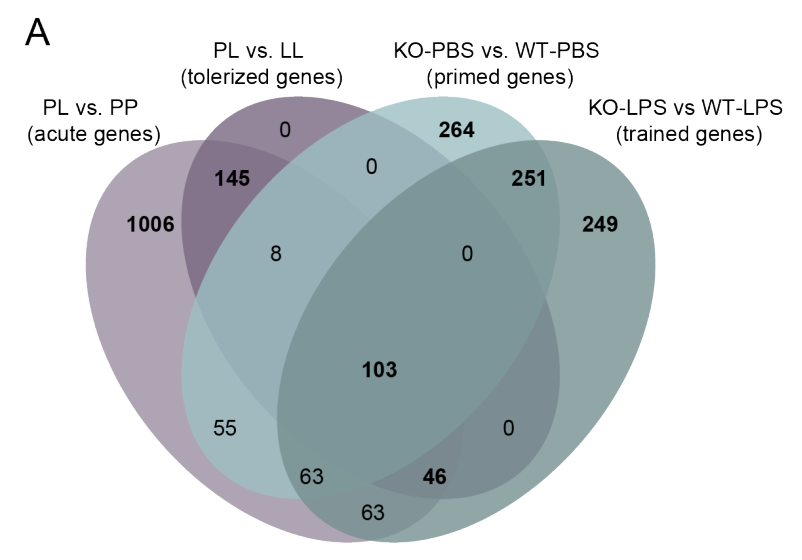

B

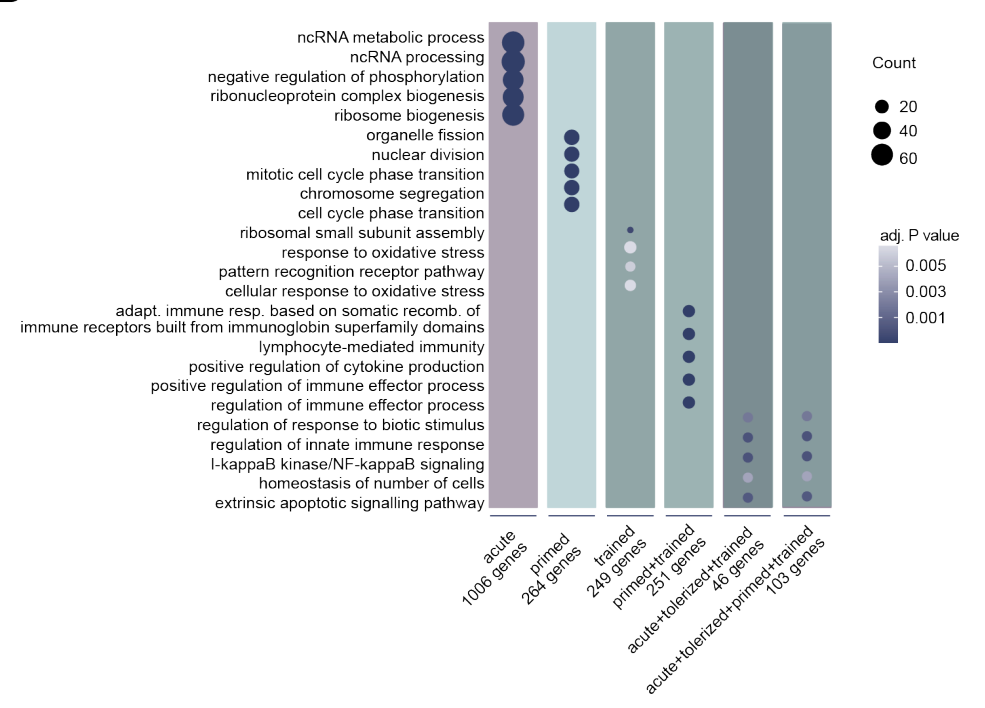

$\begin{array}{llll}C & P L \text { vs. PP } & \text { LL vs. PL } & \text { KO vs. WT } \\ \text { PBS } & \text { KO vs. WT } \\ \text { LPS }\end{array}$

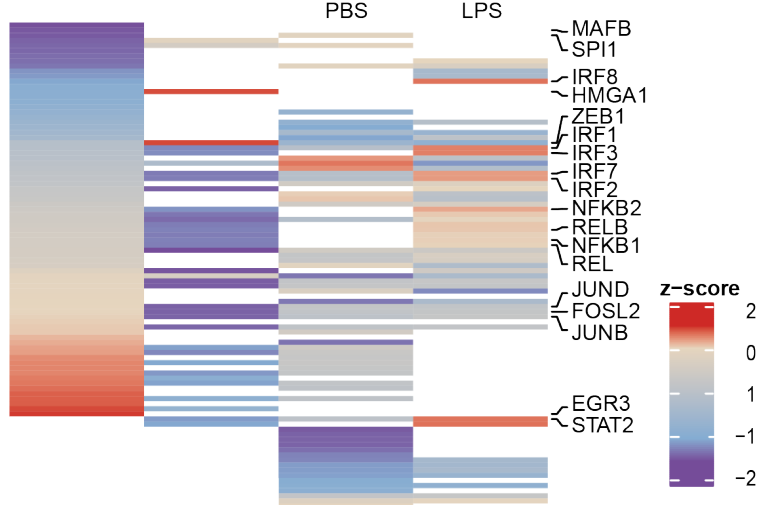

Figure 5, Inflammatory genes show distinct epigenetic regulation in 'acute', 'tolerant', 'primed' and 'trained' microglia.

a, Venn diagram of the enriched genes in acute (PL versus PP, light purple, Supplemental file 1), tolerized (clusters 2 and 4, dark purple, Supplemental file 3), primed (KO-PBS versus WTPBS, mint, Supplemental file 2) and trained (KO-LPS versus WT-LPS, dark mint, Supplemental file 2) microglial response.

b, Dotplot depicting the GO terms associated with unique and overlapping gene sets of acute, tolerant, primed and trained microglia. The size of the dot represents the gene count per GO term and the color indicates the adjusted P-value.

c, Heat map depicting row $z$-scores of weighted mean differences (adjusted $P$ value $<0.001$ ) of ATAC peaks at loci of specific TF motifs in the indicated comparisons identified with diffTF (based on Figures 3B, 3D, 4C, 4D, Supplemental file 6, 8). Only accessible TF motifs of activating, repressing and undetermined TF are displayed (not-expressed TFs were filtered out). White space indicates non-significant weighted mean differences of ATAC peaks at the given locus and/or not-expressed TFs in the indicated comparison. 
bioRxiv preprint doi: https $/ /$ doi org/10.1101/2021.05.30.446351; this version posted May 31,2021 . The copyright holder for this preprint (which was not certified by peer review) is the author/funder, who has granted bioRxiv a license to display the preprint in perpetuity. It is made available under aCC-BY 4.0 International license.

\section{Supplementary figure legends}

A
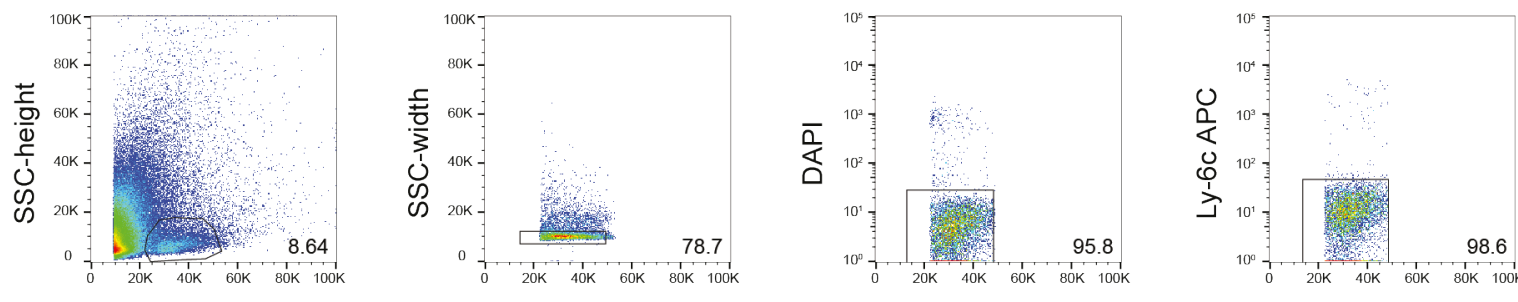

FSC1-height

B

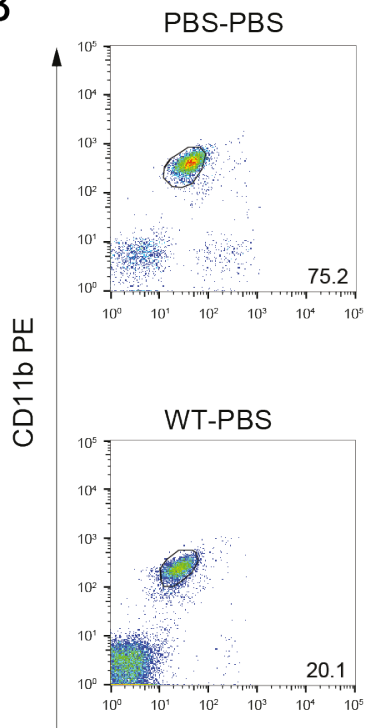

PBS-LPS

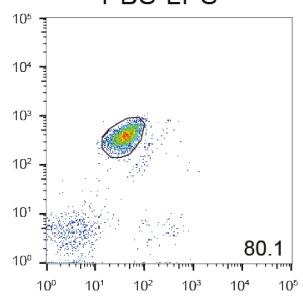

WT-LPS

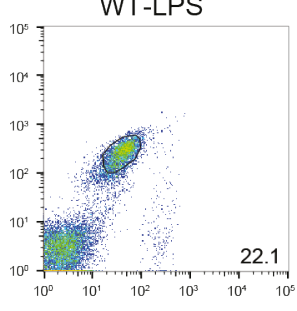

LPS-PBS

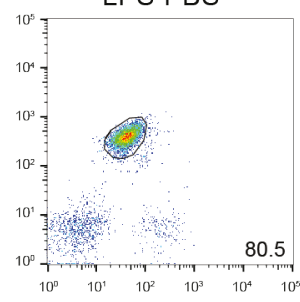

KO-PBS

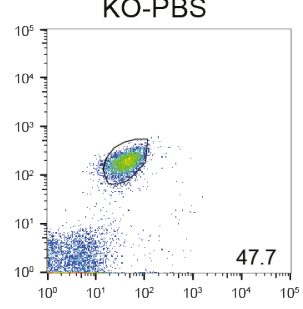

LPS-LPS

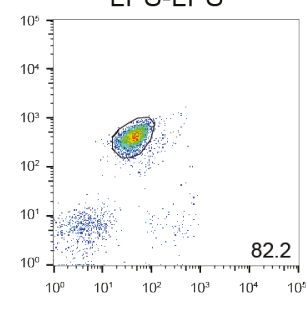

KO-LPS

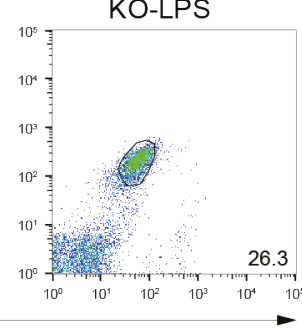

CD45 FITC

Figure S1 related to figure 1, FACS sorting of microglia.

596 a, Single, viable microglia are isolated using side scatter and forward scatter parameters, followed by exclusion of DAPIpos (dead) events. Further purification was done by exclusion of Ly-6C ${ }^{\text {pos }}$ CNS macrophages. b, CD11 ${ }^{\text {pos }}$ and CD45 int microglia were sorted. 
A

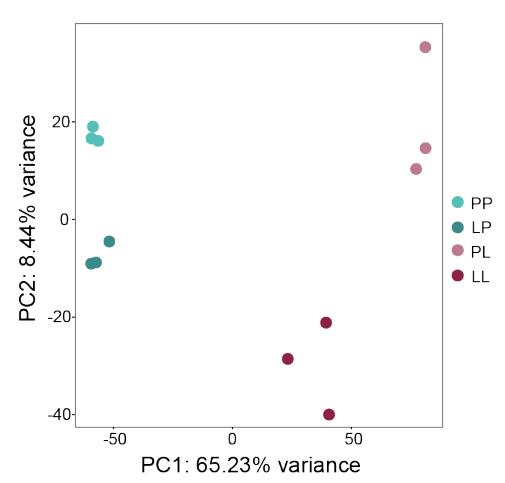

B

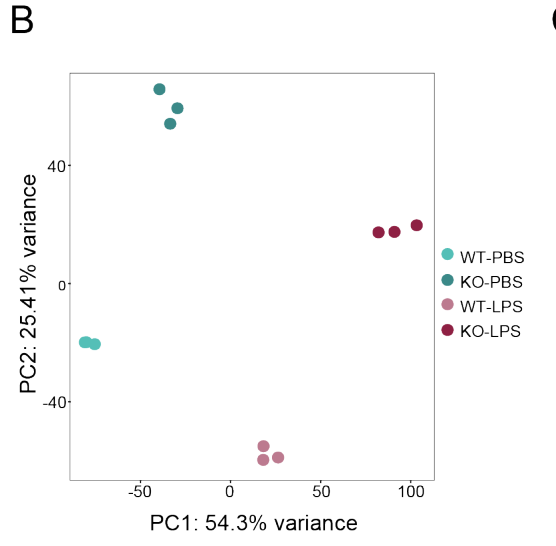

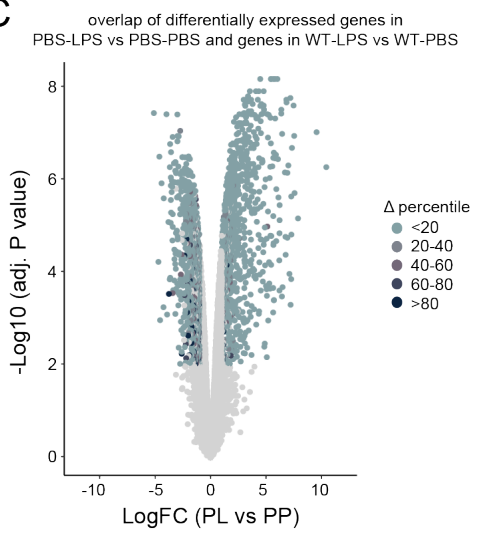

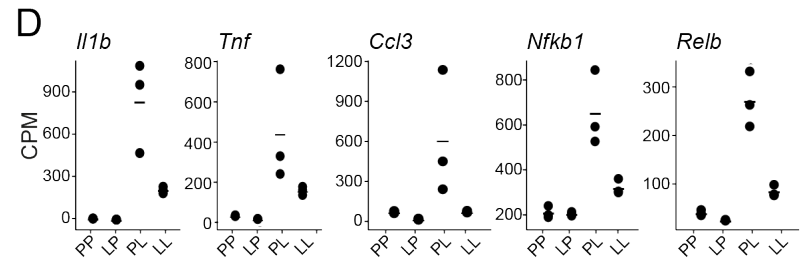
microglia.

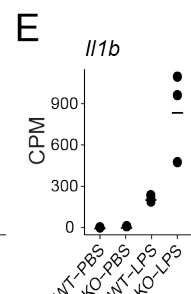

C

-

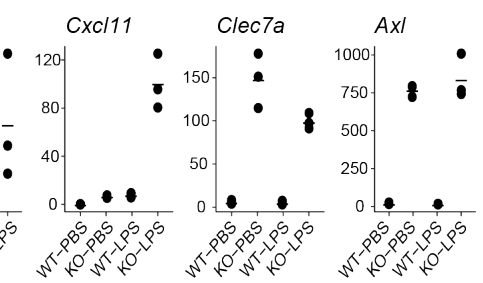

Figure $\mathbf{S 2}$ related to figure $1, \mathrm{RNA}$-sequencing of desensitized/tolerant and primed/trained

$\mathbf{a}, \mathbf{b}$, PCA-plots of RNA-seq data of microglia in the LPS desensitization tolerance (a) and Ercc1-induced priming (b) mouse models. Every dot depicts an individual animal $(n=3$ per experimental condition). c, Volcano plots illustrating the similarity in the acute LPS response in microglia from naive mice. Dots represent log fold change (LogFC) of differential expressed genes between PL and PP (c). Genes were ranked according to their expression level and based on that classified into percentiles. Next, for each gene of the two comparisons, the delta percentile was calculated and indicated as colors in the volcano plot, where light blue indicates similar and dark blue indicates deviant expression between the indicated conditions. Gray dots indicate gene expression differences with $\log F C<1$ and adjusted $P$ values $>0.01$. d, e, Gene expression values (CPM) of genes in the tolerance (d) or priming (e) mouse model. Every dot depicts an individual animal ( $n=3$ per experimental condition). CPM= counts per million reads 
bioRxiv preprint doi: https://doi.org/10.1101/2021.05.30.446351; this version posted May 31, 2021. The copyright holder for this preprint (which was not certified by peer review) is the author/funder, who has granted bioRxiv a license to display the preprint in perpetuity. It is made available under aCC-BY 4.0 International license.

A

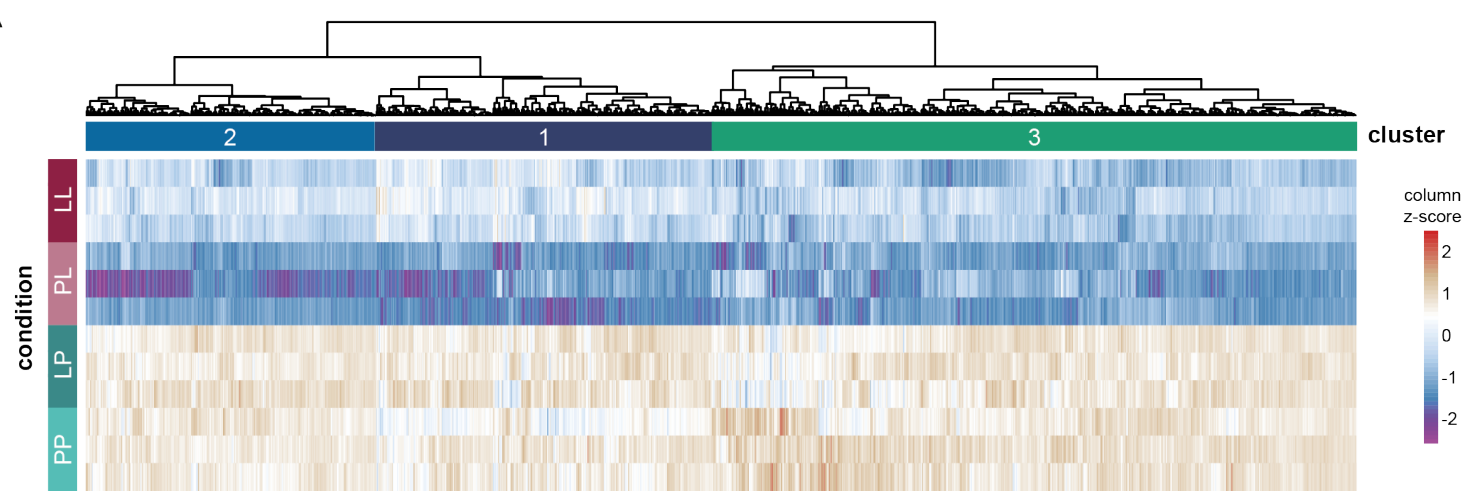

B
C top20 GO: downregulated genes by LPS regulation of defense response regulation of cytokine production regulation of cell adhesion regulation of cell activation positive regulation of programmed cell death positive regulation of locomotion positive regulation of immune response positive regulation of cytokine production positive regulation of cellular component movement positive regulation of cell motility positive regulation of apoptotic process negative regulation of cell proliferation lymphocyte activation leukocyte differentiation innate immune response inflammatory response defense response to other organism blood vessel development

$$
\begin{array}{r}
\text { temperature homeostasis } \\
\text { sulfur compound metabolic process } \\
\text { regulation of Wnt signaling pathway } \\
\text { regulation of protein serine/threonine kinase activity } \\
\text { regulation of muscle system process } \\
\text { potassium ion transport } \\
\text { positive regulation of autophagy } \\
\text { negative regulation of response to external stimulus } \\
\text { negative regulation of cell growth } \\
\text { monocarboxylic acid metabolic process } \\
\text { hexose metabolic process } \\
\text { G protein-coupled receptor signaling pathway, } \\
\text { coupled to cyclic nucleotide second messenger } \\
\text { fatty acid metabolic process } \\
\text { ear development } \\
\text { cofactor metabolic process } \\
\text { coenzyme metabolic process }
\end{array}
$$

Figure S3 related to figure 2, LPS-downregulated genes and associated GO terms.

a, Heatmap with Manhattan distance-based hierarchical clustering analysis of downregulated genes in response to LPS in microglia of C57BL/6 mice ( $n=3$ per experimental condition) three hours after i.p. injection with LPS (LogFC $>1$ and FDR $<0.01$, PP versus $P L)$. b, c, Gene ontology (GO) analysis of genes upregulated (b) and downregulated (c) 3 hours after LPS challenge in microglia of C57BL/6 mice. Based on gene count per $\mathrm{GO}$ term, the top $20 \mathrm{GO}$ terms were identified. The size of the dot represents the gene count per $\mathrm{GO}$ term and the color indicates the adjusted $P$ value. 
A

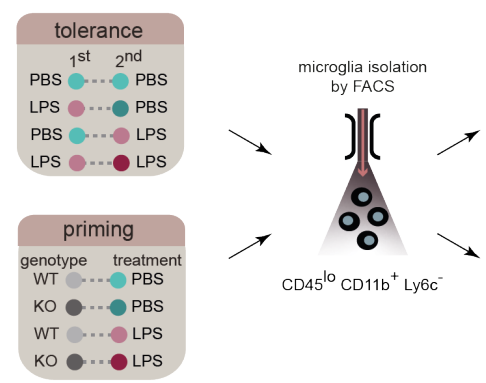

B

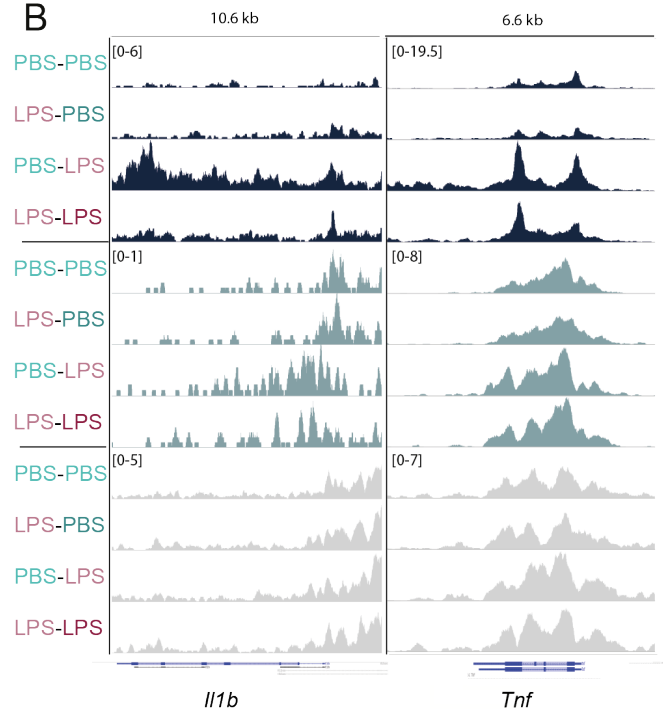

C

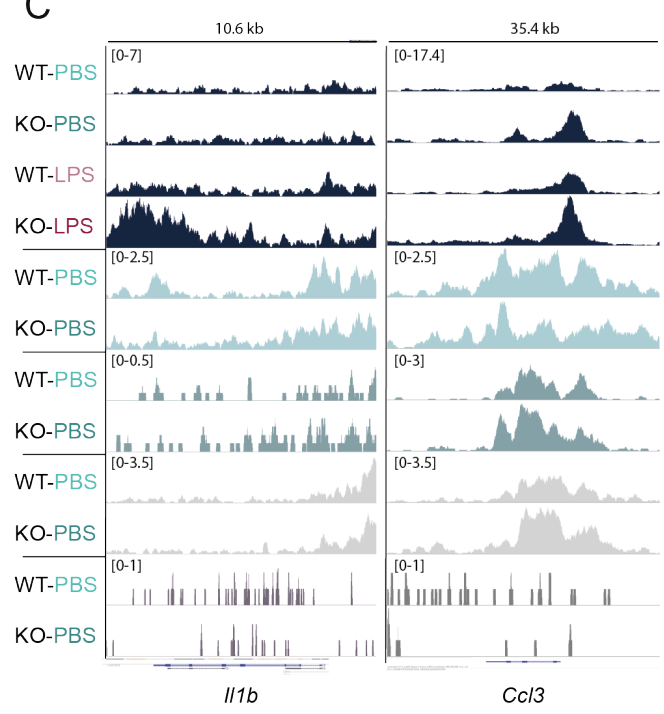

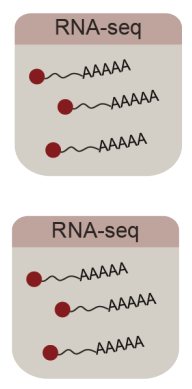
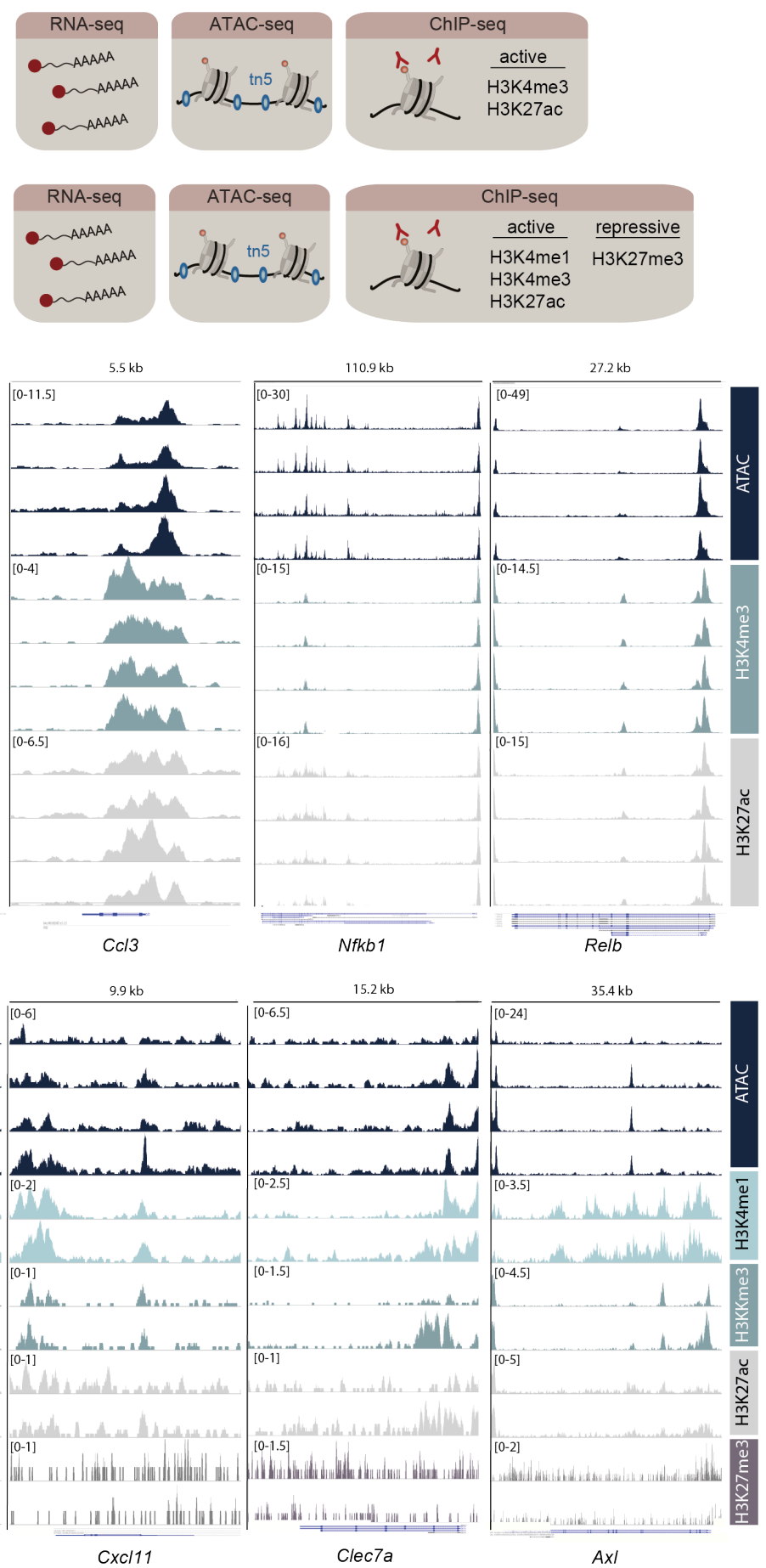

Figure S4 related to figure 3 and 4, ATAC- and ChIP-sequencing peak enrichment at representative desensitized and primed gene loci.

a, Experimental strategy for the analysis of chromatin accessibility, and occupation by histone modifications. H3K4me3 and H3K27ac were analyzed in 'desensitized' and 'tolerant' microglia. H3K4me1, H3K4me3 and H3K27ac were determined in 'primed' microglia. b, c, Tracks of ATAC and indicated histone marks sequencing data of representative desensitized/tolerant (b) and primed/trained (c) genes. For ChIP, chromatin of 5 mice per experimental group was pooled; for ATAC, microglia (80,000 total) from 2 mice per experimental group were pooled. Tracks were visualized using JetBrains SPAN peak analyzer. Gene expression of these genes are shown in S2D and S2E. 


\section{Supplementary files}

656 Supplementary file 1: count table and differentially expressed genes for all comparisons in 657 the tolerance mouse model (related to Figure 1 and 3).

658 Supplementary file 2: count table and differentially expressed genes for all comparisons in 659 the primed mouse model (related to Figure 1 and 4).

660 Supplementary file 3: Genes and associated gene ontology terms for each cluster identified 661 in the tolerance model (related to figures 2 and S3).

662 Supplementary file 4: Genes and associated gene ontology terms for each cluster identified 663 in the priming mouse model (related to figure 2 and S3).

664 Supplemental file 5: Annotated differential ATAC, H3K4me3 and H3K27ac peaks in microglia 665 of $P L$ versus $P P, P L$ versus $L L$ and $L L$ versus $L P$ mice (related to figure $3 A, 3 B, 3 C$ ).

666 Supplemental file 6: TFBS of differential ATAC peaks in microglia of PP versus PL, LL versus 667 PL and LP versus LL mice and classification of TFs based on correlation of TFBS peaks with 668 TF target gene expression (related to figure 3D, 3E, 3F, 5C).

669 Supplemental file 7: Annotated differential ATAC, H3K4me1, H3K4me3, H3K27ac and 670 H3K27ac peaks in microglia of KO-PBS versus WT-PBS, KO-LPS versus WT-LPS, WT-LPS 671 versus WT-PBS and KO-LPS versus WT-PBS mice (related to figure 4A, 4B).

672 Supplemental file 8: TFBS of differential ATAC peaks in microglia of WT-PBS versus KO673 PBS and WT-LPS versus KO-LPS mice and classification of TFs based on correlation of TFBS 674 peaks with TF target gene expression (related to Figure 4D, 4E, 5C).

675 Supplemental file 9: Lists of genes uniquely or overlappingly enriched in acute, tolerized, 676 primed and trained microglia (related to figure $5 \mathrm{~A}$ ).

677 Supplemental file 10: GO terms associated with genes uniquely or overlappingly enriched in 678 acute, tolerized, primed and trained microglia (related to figure 5B). 


\section{Methods}

\section{Animals}

681 All the animal work was performed in the Central Animal Facility of the UMCG (CDP, 682 Groningen, the Netherlands) and all animal-related studies were reviewed and approved by 683 the Animal Care and Use Committee of the University of Groningen. Animals were 684 conventionally housed under a 12/12 h light/dark cycle (8 p.m. lights off, 8 a.m. lights on) with ad libitum access to food and water.

Tolerance induction

Male C57BL/6J mice were obtained at the age of 7-9 weeks with weights in the range of 2530 grams (Envigo, Horst, the Netherlands). Upon arrival, a minimum acclimatization time of 2 weeks was ensured, where mice were monitored weekly. All animals were housed individually and randomly assigned to experimental conditions.

To induce endotoxin tolerance, mice received $1 \mathrm{mg} / \mathrm{kg}$ body weight LPS (Sigma-Aldrich, $E$. coli 0111:B4, L4391) diluted in dPBS (Lonza, BE17512F) to a total volume of $200 \mu \mathrm{L}$ by intraperitoneal injection. Immediately following LPS administration, mice were housed in a monitored daily until the body weight was completely restored (usually within 7 days). All control mice received $200 \mu \mathrm{L}$ dPBS by intraperitoneal injection. After 4 weeks, the mice received a second injection with either dPBS or LPS $(1 \mathrm{mg} / \mathrm{kg}$ body weight, diluted in $200 \mu \mathrm{L}$ dPBS).

\section{Obtaining primed microglia}

Ercc1 transgenic mice (Weeda et al., 1997) were bred in house by crossing Ercc $1^{w / t^{*} 292}$ mice (FVB background, the *292 allele is hereafter indicated with $\Delta$ ) with Ercc1wt/ko mice (BL6 background) as previously described (D. D. A. Raj et al., 2014). The offspring were genotyped after weaning using the primers listed in table 1. Ercc1 $1^{\Delta / k o}$ were used as experimental mice while littermates with Ercc1 ${ }^{\text {wt/ }}$, Ercc1 ${ }^{\text {wt/ko }}$ or Ercc1 ${ }^{\text {wtwt }}$ genotypes were used as control. All the mice were group-housed in conventional cages. Initially, mice were monitored weekly, which increased to twice per week after the aging-related symptoms appeared. Bottles with long drinking spouts were provided to prevent dehydration of $E r c c 1^{\Delta / k o}$ animals. At 11-12 weeks of age, the mice received $1 \mathrm{mg} / \mathrm{kg}$ body weight LPS or dPBS as described above. Immediately following LPS administration, mice were temporarily housed in a recovery cabinet at $26{ }^{\circ} \mathrm{C}$.

All animals were sacrificed under deep anesthesia (4\% isoflurane with $7.5 \% \mathrm{O}_{2}$ ) and perfused with cold dPBS exactly three hours after the last injection.

\section{Microglia isolation and flow cytometry}

714 Microglia were isolated as previously described (Galatro, Vainchtein, et al., 2017). After 715 perfusion, brains were removed from the skull and kept in cold medium A (HBSS (Gibco, 
14170-088) with 0.6\% glucose (Sigma, G8769) and 7.5 mM HEPES (Lonza, BE17-737E)). All subsequent steps were performed on ice, centrifugation was at $4^{\circ} \mathrm{C}$. Brains were dissociated using a potter-elvehjem tissue homogenizer after which the homogenate was passed over a $70 \mu \mathrm{M}$ cell strainer (Corning, 352350) and pelleted by centrifugation at $220 \mathrm{xg}$ for $10 \mathrm{~min}$. Next, myelin was removed by resuspending the pellet in $25 \mathrm{~mL} 24 \%$ percoll (Fisher, 17-0891-01) in medium A ( $1 \mathrm{x}$ final concentration) with $3 \mathrm{~mL}$ PBS layered on top, followed by centrifugation for $20 \mathrm{~min}$ at $950 \mathrm{xg}$ (acceleration 4 and brake 0 ). The microglia enriched cell pellets were incubated with CD11b-PE (clone M1/70, eBiosciences, 12-0112-82), CD45-FITC (clone 30F11, eBiosciences, 11-0451-82), and Ly6c-APC (clone HK1.4, Biolegend, 128016) antibodies for $30 \mathrm{~min}$ on ice. Then the cells were washed once in medium A without phenol red and filtered into FACS tubes. Microglia were sorted by gating the DAPInegCD $11 b^{\text {high }}$ CD $45^{\text {int }} L y 6 c^{\text {neg }}$ cells using the Beckman Coulter MoFlo Astrios or XDP. Microglia were collected in siliconized Eppendorf tubes (Sigma, T3406-250EA) containing medium A. Flow cytometry data was analyzed using FlowJo Analysis Software.

730

\section{RNA isolation and RNA sequencing}

732 Total RNA was isolated using a Qiagen RNeasy Micro Kit (Qiagen, 74004) according to the 733 manufacturer's instructions. cDNA was synthesized using random hexamer primers (Thermo, 734 SO142), dNTPs (Thermo, R0192) and M-MuLV Reverse Transcriptase (Thermo, EP0442) in 735 the presence of Ribolock RNase inhibitor (Thermo, EO0382). Quantitative PCR reactions were 736 performed using iTaq mastermix (Biorad, 172-5125) on ABI7900RH or QuantStudio 7 (Applied 737 Biosystems) or LightCycler ${ }^{\circledR} 480$ (Roche) PCR systems.

The quality of the total RNA was determined using an Experion (Biorad). All included samples had an RNA quality indicator $>6$. Sequencing libraries were generated with a TruSeq RNA library prep kit (Illumina, RS-122-2001). Pooled libraries were sequenced with a HiSeq Rapid SBS kit (50 cycles, Illumina, FC-402-4022) using single reads on a HiSeq 2500 (Illumina).

744 The quality of total RNA samples isolated from Ercc1 ${ }^{\Delta / k o}$ mice was determined on a LabchipGX

745 (PerkinElmer). All included samples had an RNA quality score $>5$. Sequencing libraries were 746 generated using NEXTflex ${ }^{\circledR}$ Rapid Illumina Directional RNA-Seq Library Prep Kit 747 (BiooScientific, NOVA-5138-10) with polyA selection. Pooled libraries were sequenced using 748 the NextSeq 500/550 High Output v2 kit (75 cycles, Illumina, FC-404-2005) with single reads 749 on a NextSeq500 (Illumina).

\section{RNA-sequencing analysis}


Samples ( $n=3$ per condition) were processed using our in-house pipeline, where quality control was performed with FastQC (v0.11.8). Adapter sequences and were removed with bbduk (v38.69). The Ensembl genome Mus musculus (GRCm38.82) was used for alignment (STAR v2.7.3a, Dobin et al., 2013). Sorting of the aligned reads was done with bamsort tool from biobambam2 tools (v2.0.95). featureCounts (v2.0.0, Liao et al., 2014) was used to quantify the reads. Picard (v1.130, "Picard Toolkit.," 2019) and samtools were used to perform the quality control check and the generation of the fastq files. Downstream analyses were performed using R/Bioconductor packages (v.3.11), as briefly summarized. Specific functions from EdgeR (v3.30.3, Robinson et al., 2009) were used for data normalization and calculation of rpkm expression values. Log2-CPM values and mean-variance relationship were calculated with the voomWithQualityWeights function from limma (v.3.44.3, Ritchie et al., 2015). Unwanted/hidden sources of variation were removed using sva (v3.36.0, Leek et al., 2020). Differential expression analysis was performed using limma. Annotation was performed with biomaRt (Durinck et al., 2009). For plotting purposes, genes with a $\log F C>1$ and FDR $<0.01$ were considered differentially expressed.

Clustering analysis were performed using the ward.D2 clustering method and Manhattan distance as clustering metrics. Heat maps were assembled using the pheatmap package (v1.0.12, Kolde, 2019). The optimal number of gene clusters was estimated upon visual inspection of the heat maps. Gene ontology enrichment analysis of the gene clusters was performed with the 'enrichGO' function of the clusterProfiler package (v3.16.1, Yu et al., 2012). PCA plots, scatterplots and dotplots were made with the package ggplot2 (v3.3.2, Wickham et al., 2016) and standard plot functions from R.

To visualize the overlap of differentially expressed genes of different comparisons (Figure S2C), gene lists of the indicated comparisons were ranked based on expression level. Following, percentiles were assigned to the ranked genes and $\Delta$ percentiles were calculated by subtracting percentiles of each gene from the two indicated comparisons. The results were depicted in a volcano plot, where the dots are differential expressed genes (logF>1, FDR $<0.01)$ in the indicated comparison and the color of the dots shows overlap of gene expression levels from the two indicated comparisons.

For the Venn diagram in Figure 5a, only upregulated genes of the PL versus PP, KOPBS versus WT-PBS and KO-LPs versus WT-LPS differential gene lists (Supplementary file 1 and 2) and genes of cluster 2 and 4 of the tolerance moue model (Supplementary file 3) were use. The Venn diagram was made with the 'venn' function of the gplots package (v3.1.0, Warnes et al., 2015).

\section{ChIP-sequencing}


The procedure of Chromatin immunoprecipitation has been described previously (Schaafsma et al., 2015). Sorted microglia were fixed in $1 \mathrm{~mL} 1 \%$ formaldehyde diluted in $\mathrm{dPBS}$ at $20^{\circ} \mathrm{C}$ for $10 \mathrm{~min}$ and fixation was stopped by adding glycine to a final concentration of $0.125 \mathrm{M}$ glycine. Fixed cells were washed twice by $1 \mathrm{~mL}$ cold dPBS, and then lysed with cell lysis buffer $(5 \mathrm{mM}$ Pipes, pH8.0; $85 \mathrm{mM} \mathrm{KCl} ; 0.5 \% \mathrm{NP}-40$ ) by incubating on ice for $10 \mathrm{~min}$. At the end, the cells were lysed in $250 \mu \mathrm{L}$ nuclear lysis buffer (50 mM Tris. $\mathrm{HCl}, \mathrm{pH} 8.1 ; 10 \mathrm{mM}$ EDTA, pH8.0; 1\% SDS) to obtain the crosslinked chromatin. Chromatin was sonicated using a Bioruptor (Diagenode) at "high" power for $20 \mathrm{~min}$ (30 sec on and $30 \mathrm{sec}$ off, for 20 cycles) at $4^{\circ} \mathrm{C}$. Chromatin from animals within the same treatment group was pooled ( 5 animals per pool) and precleared using protein A agarose beads (25\%, diluted in ChIP dilution buffer; Protein A Agarose/Salmon Sperm DNA, Millipore, 16-157) and incubated overnight at $4^{\circ} \mathrm{C}$ (final buffer composition during antibody incubation was $0.1 \%$ SDS; $1 \%$ Triton-X-100; 2.4 mM EDTA; 20 mMTris. $\mathrm{HCl}, \mathrm{pH} 8.1 ; 150 \mathrm{mM} \mathrm{NaCl}$ ) with antibodies for specific histone modifications (the information of antibodies is available in table 2, the specificity of antibodies have been checked for H3K27me3 peptides, the information of these peptides is listed in table 2). The chromatin incubated with $\lg G$ was used as negative control while the chromatin saved without antibody incubation served as input. The next day, immune complexes were precipitated with $80 \mu \mathrm{L}$ protein $\mathrm{A}$ beads $(25 \%)$ for $2 \mathrm{~h}$ at $4{ }^{\circ} \mathrm{C}$, washed by low salt wash buffer $(150 \mathrm{mM} \mathrm{NaCl} ; 0.1 \%$ SDS; $1 \%$ Triton-x-100; 2 mM EDTA, pH8.0; 20 mM Tris. $\mathrm{HCl}, \mathrm{pH} 8.1$ ), high salt wash buffer (500 $\mathrm{mM} \mathrm{NaCl} ; 0.1 \%$ SDS; 1\% Triton-x-100; 2 mM EDTA, pH8.0; 20 mM Tris. $\mathrm{HCl}, \mathrm{pH} 8.1$ ), LiCl wash buffer $(0.25 \mathrm{M} \mathrm{LiCl}$; 1\% NP-40; 1\% Na-deoxycholate; 1 mM EDTA; $10 \mathrm{mM}$ Tris. $\mathrm{HCl}$, $\mathrm{pH} 8.1$ ), and TE (10 mM Tris. $\mathrm{HCl}, \mathrm{pH} 8.0 ; 1 \mathrm{mM}$ EDTA, pH8.0). After the chromatin was eluted from the beads, the precipitated chromatin was de-crosslinked overnight at $65^{\circ} \mathrm{C}$. Afterwards, RNase A (Thermofisher, EN0531) and Proteinase K (Sigma, P2308) were added. Finally, the DNA was purified by GeneJET PCR purification kit (ThermoFisher, k0701).

Sequencing libraries were generated from the purified DNA by MicroPlex Library Preparation Kit v1 x12 (Diagenode, C05010010) for tolerized samples or MicroPlex Library Preparation Kit v2 x12 (Diagenode, C05010012) in case of primed samples. The libraries were quantified by Agilent 2100 Bioanalyzer, pooled and sequenced with a HiSeq Rapid SBS kit (50 cycles, Illumina, FC-402-4022) using single reads on a HiSeq 2500 (Illumina).

\section{ATAC sequencing}

ATAC-sequencing libraries were generated using Nextera ${ }^{\circledR}$ DNA Sample Preparation Kit (Illumina, FC-121-1030) following the methods described by Buenrostro et al., 2013, 2015. A total number of 80,000 microglia were pooled from two animals $(40,000$ cells from each) and collected in Eppendorf tubes containing $300 \mu \mathrm{L}$ medium A. Cells were pelleted by centrifugation (10 $\mathrm{min}, 4^{\circ} \mathrm{C}, 500 \mathrm{xg}$ ), resuspended in $50 \mu \mathrm{L}$ of cold lysis buffer (10 mM Tris- 
$\mathrm{HCl}, \mathrm{pH} 7.4,10 \mathrm{mM} \mathrm{NaCl}, 3 \mathrm{mM} \mathrm{MgCl} 2,0.1 \%$ IGEPAL CA-630) and immediately centrifuged as before. Next, nuclei were resuspended in $50 \mu \mathrm{L}$ transposition reaction mix (1x TD reaction buffer, $2.5 \mu \mathrm{L}$ TN5 transposase) and incubated at $37^{\circ} \mathrm{C}$ for $30 \mathrm{~min}$. Immediately following transposition, the DNA was purified using a minElute PCR purification kit (Qiagen, 28004) following the manufacturer's instructions. The transposed DNA fragments were further amplified and barcoded (Buenrostro et al., 2013, 2015) and purified with a ChIP DNA Clean \& Concentrator kit (Zymo, D5205). The fragments were run on $2 \% \mathrm{E}-\mathrm{Gel}^{\mathrm{TM}} \mathrm{EX}$ agarose gels (Thermo Fisher scientific, G521802) and 150-600 bp fragments were excised, followed by purification with Zymoclean ${ }^{\mathrm{TM}}$ Gel DNA Recovery Kit (Zymo, D4007). Library concentration was determined with an Agilent 2100 Bioanalyzer after which 8 samples were pooled and sequenced using HiSeq Rapid SBS Kit v2 (50 cycles) using paired end reads on a HiSeq2500 (Illumina).

\section{ChIP- and ATAC-sequencing analysis}

ATAC and ChIP samples were aligned to the Mus musculus genome (mm10/GRCm38) with the use of Bowtie 2 (v2.3.5.1, Langmead \& Salzberg, 2012) using the -very-sensitive flag. Bamsort and bammarkduplicates from biobambam2 tools (v2.0.95) were used to sort the aligned files and to remove duplicated reads. Samtools (v.1.1.0, Danecek et al., 2021) was used to remove low quality $(q<30)$ and blacklisted alignments. For Chip-seq data, peak calling was performed using the JetBrains SPAN peak analyzer (v.0.11.0) using default parameters, which were later manually refined upon visual inspection using the JetBrains JBR browser (v.1.0 beta) on each sample. BigWig files were generated using deepTools bamCoverage (v.3.5.0) with RPGC normalization. ATAC-seq peaks were called using Genrich (v.0.5) with the ATAC-seq mode (-j switch), and -p parameter set to 0.01. Differential peak calling for ChIPand ATAC-seq were performed with manorm (v.1.3.0, Shao et al., 2012). The annotation of differential peaks was performed with the annotatePeaks function from R/Bioconductor ChIPseeker package (v1.24.0, G. Yu et al., 2015).

Analysis of differential transcription factor binding sites accessibility and classification of transcription factors into activators, repressors or undetermined was performed with the diffTF package (v1.7.1., Berest et al., 2019) based on ATAC- and RNA-seq data. The heatmap in Figure $5 c$ is based on the diffTF output in Supplemental file $6 \& 8$. The row z-score was calculated from weighted mean differences of ATAC peaks from the indicated comparison. Following, in each comparison non-significant differential peaks (adjusted $P$ value $>0.001$ ) and TF classified as 'not-expressed' were omitted. The row z-scores of significant differential accessible regions (adjusted $P$ value $>0.001$ ) of putative transcriptional 
861 activators, repressors and undetermined TFs are visualized in a heatmap assembled with the

862 'Heatmap' function of the ComplexHeatmap package (v2.4.3, Gu et al., 2016).

863 Tables

864 Table 1. Ercc1 genotyping primers

\begin{tabular}{llll}
\hline allele & product size & forward primer 5'-3' $^{\prime}$ & reverse primer 5'-3' \\
\hline$W T$ & $246 \mathrm{bp}$ & AGCCGACCTCCTTATGGAAA & ACAGATGCTGAGGGCAGACT \\
$K O$ & $390 \mathrm{bp}$ & TCGCCTTCTTGACGAGTTCT & ACAGATGCTGAGGGCAGACT \\
$292^{*}(\Delta)$ & $530 \mathrm{bp}$ & TCGCCTTCTTGACGAGTTCT & CTAGGTGGCAGCAGGTCATC \\
\hline
\end{tabular}

865

866 Table 2. Antibodies used for ChIP

\begin{tabular}{lllll}
\hline antibody & supplier & full name & cat \# & lot \# \\
\hline H3K4me1 & Abcam & Anti-Histone H3 (mono methyl K4) antibody - ChIP Grade & Ab8895 & GR193737-1 \\
H3K4me3 & Millipore & Anti-trimethyl-Histone H3 (Lys4) & $07-473$ & 2117175 \\
H3K27ac & Abcam & Anti-Histone H3 (acetyl K27) antibody - ChIP Grade & Ab4729 & GR200563-1 \\
H3K27me3 & Millipore & ChIP Ab+tm Trimethyl-Histone H3 (Lys27) & $17-622$ & 2325081 \\
\hline
\end{tabular}




\section{Acknowledgements}

869 The authors thank Karina Hoekstra-Wakker, Nancy Halsema, and Diana Spierings for

870 sequencing support, Geert Mesander, Henk Moes, and Roelof Jan van der Lei for technical

871 assistance with FACS sorting, and Hilmar RJ van Weering for artwork. This work was

872 supported by a China Scholarship Council fellowship to XZ (Grant \# 201306300082) and a

873 Graduate School of Medical Sciences-UMCG scholarship to LK. SMK is funded by the

874 Netherlands Organization for Scientific Research (NWO, VENI, \#016.161.072) and the MS

875 Research Foundation (16-947).

876

877

\section{Author contributions}

879 Conceptualization, X.Z., S.M.K., B.J.L.E.; Formal Analysis, A.M.L., M.L.D., L.K.; Investigation, 880 X.Z., S.M.K., L.K., N.B., E.M.W.; Writing - Original Draft, S.M.K., B.J.L.E.; Writing - Review \& 881 Editing, X.Z., L.K., B.J.L.E., S.M.K., A.M.L., M.L.D., N.B., E.M.W., H.W.G.M.B.; Supervision, 882 S.M.K., B.J.L.E.; Funding Acquisition, X.Z., S.M.K., H.W.G.M.B., B.J.L.E.

883

884

\section{Declaration of interests}

886 The authors declare no competing interests.

887

888

889

\section{Data availability statement}

890 All next-generation sequencing data can be viewed at NCBI GEO under accession number 891 GSE175578. 
893

894

895

896

897

898

899

900

901

902

903

904

905

906

907

908

909

910

911

912

913

914

915

916

917

918

919

920

921

922

923

924

925

\section{References}

Arts, R. J. W., Joosten, L. A. B., \& Netea, M. G. (2018). The potential role of trained immunity in autoimmune and autoinflammatory disorders. In Frontiers in Immunology (Vol. 9, Issue FEB). Frontiers Media S.A. https://doi.org/10.3389/fimmu.2018.00298

Arts, R. J. W., Moorlag, S. J. C. F. M., Novakovic, B., Li, Y., Wang, S. Y., Oosting, M., Kumar, V., Xavier, R. J., Wijmenga, C., Joosten, L. A. B., Reusken, C. B. E. M., Benn, C. S., Aaby, P., Koopmans, M. P., Stunnenberg, H. G., van Crevel, R., \& Netea, M. G. (2018). BCG Vaccination Protects against Experimental Viral Infection in Humans through the Induction of Cytokines Associated with Trained Immunity. Cell Host and Microbe, 23(1), 89-100.e5. https://doi.org/10.1016/j.chom.2017.12.010

Askew, K., Li, K., Olmos-Alonso, A., Garcia-Moreno, F., Liang, Y., Richardson, P., Tipton, T., Chapman, M. A., Riecken, K., Beccari, S., Sierra, A., Molnár, Z., Cragg, M. S., Garaschuk, O., Perry, V. H., \& Gomez-Nicola, D. (2017). Coupled Proliferation and Apoptosis Maintain the Rapid Turnover of Microglia in the Adult Brain. Cell Reports, 18(2), 391-405. https://doi.org/10.1016/j.celrep.2016.12.041

Ayata, P., Badimon, A., Strasburger, H. J., Duff, M. K., Montgomery, S. E., Loh, Y. H. E., Ebert, A., Pimenova, A. A., Ramirez, B. R., Chan, A. T., Sullivan, J. M., Purushothaman, I., Scarpa, J. R., Goate, A. M., Busslinger, M., Shen, L., Losic, B., \& Schaefer, A. (2018). Epigenetic regulation of brain region-specific microglia clearance activity. Nature Neuroscience, 21(8), 1049-1060. https://doi.org/10.1038/s41593-018-0192-3

Berest, I., Arnold, C., Reyes-Palomares, A., Palla, G., Rasmussen, K. D., Giles, H., Bruch, P. M., Huber, W., Dietrich, S., Helin, K., \& Zaugg, J. B. (2019). Quantification of Differential Transcription Factor Activity and Multiomics-Based Classification into Activators and Repressors: diffTF. Cell Reports, 29(10), 3147-3159.e12. https://doi.org/10.1016/j.celrep.2019.10.106

Biswas, S. K., \& Lopez-Collazo, E. (2009). Endotoxin tolerance: new mechanisms, molecules and clinical significance. In Trends in Immunology (Vol. 30, Issue 10, pp. 475-487). Trends Immunol. https://doi.org/10.1016/j.it.2009.07.009

Block, C. J., Dyson, G., Campeanu, I. J., Watza, D., Ratnam, M., \& Wu, G. (2019). A stromacorrected ZEB1 transcriptional signature is inversely associated with antitumor immune activity in breast cancer. Scientific Reports, 9(1), 1-14. https://doi.org/10.1038/s41598019-54282-z

Block, M. L., Zecca, L., \& Hong, J. S. (2007). Microglia-mediated neurotoxicity: Uncovering 
the molecular mechanisms. Nature Reviews Neuroscience, 8(1), 57-69. https://doi.org/10.1038/nrn2038

Brady, O. A., Martina, J. A., \& Puertollano, R. (2018). Emerging roles for TFEB in the immune response and inflammation. In Autophagy (Vol. 14, Issue 2, pp. 181-189). Taylor and Francis Inc. https://doi.org/10.1080/15548627.2017.1313943

Buenrostro, J. D., Giresi, P. G., Zaba, L. C., Chang, H. Y., \& Greenleaf, W. J. (2013). Transposition of native chromatin for fast and sensitive epigenomic profiling of open chromatin, DNA-binding proteins and nucleosome position. Nature Methods, 10(12), 1213-1218. https://doi.org/10.1038/nmeth.2688

Buenrostro, J. D., Wu, B., Chang, H. Y., \& Greenleaf, W. J. (2015). ATAC-seq: A method for assaying chromatin accessibility genome-wide. Current Protocols in Molecular Biology, 2015, 21.29.1-21.29.9. https://doi.org/10.1002/0471142727.mb2129s109

Butovsky, O., Jedrychowski, M. P., Moore, C. S., Cialic, R., Lanser, A. J., Gabriely, G., Koeglsperger, T., Dake, B., Wu, P. M., Doykan, C. E., Fanek, Z., Liu, L., Chen, Z., Rothstein, J. D., Ransohoff, R. M., Gygi, S. P., Antel, J. P., \& Weiner, H. L. (2014). Identification of a unique TGF- $\beta$-dependent molecular and functional signature in microglia. Nature Neuroscience, 17(1), 131-143. https://doi.org/10.1038/nn.3599

Butovsky, O., \& Weiner, H. L. (2018). Microglial signatures and their role in health and disease. In Nature Reviews Neuroscience (Vol. 19, Issue 10, pp. 622-635). Nature Publishing Group. https://doi.org/10.1038/s41583-018-0057-5

Cheng, S. C., Quintin, J., Cramer, R. A., Shepardson, K. M., Saeed, S., Kumar, V., Giamarellos-Bourboulis, E. J., Martens, J. H. A., Rao, N. A., Aghajanirefah, A., Manjeri, G. R., Li, Y., Ifrim, D. C., Arts, R. J. W., Van Der Meer, B. M. J. W., Deen, P. M. T., Logie, C., O'Neill, L. A., Willems, P., ... Netea, M. G. (2014). MTOR- and HIF-1amediated aerobic glycolysis as metabolic basis for trained immunity. Science, 345(6204). https://doi.org/10.1126/science.1250684

Cho, S. H., Chen, J. A., Sayed, F., Ward, M. E., Gao, F., Nguyen, T. A., Krabbe, G., Sohn, P. D., Lo, I., Minami, S., Devidze, N., Zhou, Y., Coppola, G., \& Gan, L. (2015). SIRT1 deficiency in microglia contributes to cognitive decline in aging and neurodegeneration via epigenetic regulation of IL-1 $\beta$. Journal of Neuroscience, 35(2), 807-818. https://doi.org/10.1523/JNEUROSCI.2939-14.2015

Cooper-Knock, J., Green, C., Altschuler, G., Wei, W., Bury, J. J., Heath, P. R., Wyles, M., Gelsthorpe, C., Highley, J. R., Lorente-Pons, A., Beck, T., Doyle, K., Otero, K., Traynor, 
959

960

961

962

963

964

965

966

967

968

969

970

971

972

973

974

975

976

977

978

979

980

981

982

983

984

985

986

987

988

989

990

B., Kirby, J., Shaw, P. J., \& Hide, W. (2017). A data-driven approach links microglia to pathology and prognosis in amyotrophic lateral sclerosis. Acta Neuropathologica Communications, 5(1), 23. https://doi.org/10.1186/s40478-017-0424-x

Creyghton, M. P., Cheng, A. W., Welstead, G. G., Kooistra, T., Carey, B. W., Steine, E. J., Hanna, J., Lodato, M. A., Frampton, G. M., Sharp, P. A., Boyer, L. A., Young, R. A., \& Jaenisch, R. (2010). Histone H3K27ac separates active from poised enhancers and predicts developmental state. Proceedings of the National Academy of Sciences of the United States of America, 107(50), 21931-21936.

https://doi.org/10.1073/pnas.1016071107

Cunningham, C., Wilcockson, D. C., Campion, S., Lunnon, K., \& Perry, V. H. (2005). Central and systemic endotoxin challenges exacerbate the local inflammatory response and increase neuronal death during chronic neurodegeneration. Journal of Neuroscience, 25(40), 9275-9284. https://doi.org/10.1523/JNEUROSCI.2614-05.2005

Danecek, P., Bonfield, J. K., Liddle, J., Marshall, J., Ohan, V., Pollard, M. O., Whitwham, A., Keane, T., McCarthy, S. A., Davies, R. M., \& Li, H. (2021). Twelve years of SAMtools and BCFtools. GigaScience, 10(2). https://doi.org/10.1093/gigascience/giab008

Dobin, A., Davis, C. A., Schlesinger, F., Drenkow, J., Zaleski, C., Jha, S., Batut, P., Chaisson, M., \& Gingeras, T. R. (2013). STAR: Ultrafast universal RNA-seq aligner. Bioinformatics, 29(1), 15-21. https://doi.org/10.1093/bioinformatics/bts635

Durinck, S., Spellman, P. T., Birney, E., \& Huber, W. (2009). Mapping identifiers for the integration of genomic datasets with the R/ Bioconductor package biomaRt. Nature Protocols, 4(8), 1184-1191. https://doi.org/10.1038/nprot.2009.97

Eggen, B. J.L., Raj, D., Hanisch, U. K., \& Boddeke, H. W. G. M. (2013). Microglial phenotype and adaptation. In Journal of Neuroimmune Pharmacology (Vol. 8, Issue 4, pp. 807823). https://doi.org/10.1007/s11481-013-9490-4

Eggen, Bart J.L., Boddeke, E. W. G. M., \& Kooistra, S. M. (2017). Regulation of Microglia Identity from an Epigenetic and Transcriptomic Point of View. In Neuroscience (Vol. 405, pp. 3-13). Elsevier Ltd. https://doi.org/10.1016/j.neuroscience.2017.12.010

El Gazzar, M., Yoza, B. K., Chen, X., Hu, J., Hawkins, G. A., \& McCall, C. E. (2008). G9a and HP1 couple histone and DNA methylation to TNF $\alpha$ transcription silencing during endotoxin tolerance. Journal of Biological Chemistry, 283(47), 32198-32208. https://doi.org/10.1074/jbc.M803446200 
Escoubet-Lozach, L., Benner, C., Kaikkonen, M. U., Lozach, J., Heinz, S., Spann, N. J., Crotti, A., Stender, J., Ghisletti, S., Reichart, D., Cheng, C. S., Luna, R., Ludka, C., Sasik, R., Garcia-Bassets, I., Hoffmann, A., Subramaniam, S., Hardiman, G., Rosenfeld, M. G., \& Glass, C. K. (2011). Mechanisms establishing tlr4-responsive activation states of inflammatory response genes. PLoS Genetics, 7(12). https://doi.org/10.1371/journal.pgen.1002401

Estes, M. L., \& McAllister, A. K. (2016). Maternal immune activation: Implications for neuropsychiatric disorders. In Science (Vol. 353, Issue 6301, pp. 772-777). American Association for the Advancement of Science. https://doi.org/10.1126/science.aag3194

Foster, S. L., Hargreaves, D. C., \& Medzhitov, R. (2007). Gene-specific control of inflammation by TLR-induced chromatin modifications. Nature, 447(7147), 972-978. https://doi.org/10.1038/nature05836

Füger, P., Hefendehl, J. K., Veeraraghavalu, K., Wendeln, A. C., Schlosser, C., Obermüller, U., Wegenast-Braun, B. M., Neher, J. J., Martus, P., Kohsaka, S., Thunemann, M., Feil, R., Sisodia, S. S., Skodras, A., \& Jucker, M. (2017). Microglia turnover with aging and in an Alzheimer's model via long-term in vivo single-cell imaging. Nature Neuroscience, 20(10), 1371-1376. https://doi.org/10.1038/nn.4631

Galatro, T. F., Holtman, I. R., Lerario, A. M., Vainchtein, I. D., Brouwer, N., Sola, P. R., Veras, M. M., Pereira, T. F., Leite, R. E. P., Möller, T., Wes, P. D., Sogayar, M. C., Laman, J. D., Den Dunnen, W., Pasqualucci, C. A., Oba-Shinjo, S. M., Boddeke, E. W. G. M., Marie, S. K. N., \& Eggen, B. J. L. (2017). Transcriptomic analysis of purified human cortical microglia reveals age-associated changes. Nature Neuroscience, 20(8), 1162-1171. https://doi.org/10.1038/nn.4597

Galatro, T. F., Vainchtein, I. D., Brouwer, N., Boddeke, E. W. G. M., \& Eggen, B. J. L. (2017). Isolation of microglia and immune infiltrates from mouse and primate central nervous system. In Methods in Molecular Biology (Vol. 1559, pp. 333-342). Humana Press Inc. https://doi.org/10.1007/978-1-4939-6786-5_23

Gautier, E. L., Shay, T., Miller, J., Greter, M., Jakubzick, C., Ivanov, S., Helft, J., Chow, A., Elpek, K. G., Gordonov, S., Mazloom, A. R., Ma'Ayan, A., Chua, W. J., Hansen, T. H., Turley, S. J., Merad, M., Randolph, G. J., Best, A. J., Knell, J., .. Benoist, C. (2012). Gene-expression profiles and transcriptional regulatory pathways that underlie the identity and diversity of mouse tissue macrophages. Nature Immunology, 13(11), 11181128. https://doi.org/10.1038/ni.2419 
1024

1025

1026

1027

1028

1029

1030

1031

1032

1033

1034

1035

1036

1037

1038

1039

1040

1041

1042

1043

1044

1045

1046

1047

1048

1049

1050

1051

1052

1053

1054

1055

1056

Glass, C. K., \& Natoli, G. (2016). Molecular control of activation and priming in macrophages. In Nature Immunology (Vol. 17, Issue 1, pp. 26-33). Nature Publishing Group. https://doi.org/10.1038/ni.3306

Gómez-Nicola, D., Fransen, N. L., Suzzi, S., \& Hugh Perry, V. (2013). Regulation of microglial proliferation during chronic neurodegeneration. Journal of Neuroscience, 33(6), 2481-2493. https://doi.org/10.1523/JNEUROSCI.4440-12.2013

Gosselin, D., Link, V. M., Romanoski, C. E., Fonseca, G. J., Eichenfield, D. Z., Spann, N. J., Stender, J. D., Chun, H. B., Garner, H., Geissmann, F., \& Glass, C. K. (2014). Environment drives selection and function of enhancers controlling tissue-specific macrophage identities. Cell, 159(6), 1327-1340. https://doi.org/10.1016/j.cell.2014.11.023

Gosselin, D., Skola, D., Coufal, N. G., Holtman, I. R., Schlachetzki, J. C. M., Sajti, E., Jaeger, B. N., O'Connor, C., Fitzpatrick, C., Pasillas, M. P., Pena, M., Adair, A., Gonda, D. D., Levy, M. L., Ransohoff, R. M., Gage, F. H., \& Glass, C. K. (2017). An environmentdependent transcriptional network specifies human microglia identity. Science, 356(6344), 1248-1259. https://doi.org/10.1126/science.aal3222

Gu, Z., Eils, R., \& Schlesner, M. (2016). Complex heatmaps reveal patterns and correlations in multidimensional genomic data. Bioinformatics, 32(18), 2847-2849. https://doi.org/10.1093/bioinformatics/btw313

Haley, M. J., Brough, D., Quintin, J., \& Allan, S. M. (2017). Microglial Priming as Trained Immunity in the Brain. In Neuroscience (Vol. 405, pp. 47-54). Elsevier Ltd. https://doi.org/10.1016/j.neuroscience.2017.12.039

Hammond, T. R., Dufort, C., Dissing-Olesen, L., Giera, S., Young, A., Wysoker, A., Walker, A. J., Gergits, F., Segel, M., Nemesh, J., Marsh, S. E., Saunders, A., Macosko, E., Ginhoux, F., Chen, J., Franklin, R. J. M., Piao, X., McCarroll, S. A., \& Stevens, B. (2019). Single-Cell RNA Sequencing of Microglia throughout the Mouse Lifespan and in the Injured Brain Reveals Complex Cell-State Changes. Immunity, 50(1), 253-271.e6. https://doi.org/10.1016/j.immuni.2018.11.004

Hargreaves, D. C., Horng, T., \& Medzhitov, R. (2009). Control of Inducible Gene Expression by Signal-Dependent Transcriptional Elongation. Cell, 138(1), 129-145. https://doi.org/10.1016/j.cell.2009.05.047

Henikoff, S., \& Shilatifard, A. (2011). Histone modification: Cause or cog? In Trends in Genetics (Vol. 27, Issue 10, pp. 389-396). Trends Genet. 
https://doi.org/10.1016/j.tig.2011.06.006

1058

1059

1060

1061

1062

1063

1064

1065

1066

1067

1068

1069

1070

1071

1072

1073

1074

1075

1076

1077

1078

1079

1080

1081

1082

1083

1084

1085

1086

1087

1088

1089

Hickman, S. E., Kingery, N. D., Ohsumi, T. K., Borowsky, M. L., Wang, L. C., Means, T. K., \& El Khoury, J. (2013). The microglial sensome revealed by direct RNA sequencing. Nature Neuroscience, 16(12), 1896-1905. https://doi.org/10.1038/nn.3554

Holtman, I. R., Raj, D. D., Miller, J. A., Schaafsma, W., Yin, Z., Brouwer, N., Wes, P. D., Moller, T., Orre, M., Kamphuis, W., Hol, E. M., Boddeke, E. W. G. M., \& Eggen, B. J. L. (2015). Induction of a common microglia gene expression signature by aging and neurodegenerative conditions: a co-expression meta-analysis. Acta Neuropathologica Communications, 3, 31. https://doi.org/10.1186/s40478-015-0203-5

Holtman, I. R., Skola, D., \& Glass, C. K. (2017). Transcriptional control of microglia phenotypes in health and disease. In Journal of Clinical Investigation (Vol. 127, Issue 9 , pp. 3220-3229). American Society for Clinical Investigation.

https://doi.org/10.1172/JCI90604

Keren-Shaul, H., Spinrad, A., Weiner, A., Matcovitch-Natan, O., Dvir-Szternfeld, R., Ulland, T. K., David, E., Baruch, K., Lara-Astaiso, D., Toth, B., Itzkovitz, S., Colonna, M., Schwartz, M., \& Amit, I. (2017). A Unique Microglia Type Associated with Restricting Development of Alzheimer's Disease. Cell, 169(7), 1276-1290.e17. https://doi.org/10.1016/j.cell.2017.05.018

Kierdorf, K., Erny, D., Goldmann, T., Sander, V., Schulz, C., Perdiguero, E. G., Wieghofer, P., Heinrich, A., Riemke, P., Hölscher, C., Müller, D. N., Luckow, B., Brocker, T., Debowski, K., Fritz, G., Opdenakker, G., Diefenbach, A., Biber, K., Heikenwalder, M., ... Prinz, M. (2013). Microglia emerge from erythromyeloid precursors via Pu.1-and Irf8dependent pathways. Nature Neuroscience, 16(3), 273-280.

https://doi.org/10.1038/nn.3318

Kleinnijenhuis, J., Quintin, J., Preijers, F., Joosten, L. A. B., Ifrim, D. C., Saeed, S., Jacobs, C., Van Loenhout, J., De Jong, D., Hendrik, S., Xavier, R. J., Van Der Meer, J. W. M., Van Crevel, R., \& Netea, M. G. (2012). Bacille Calmette-Guérin induces NOD2dependent nonspecific protection from reinfection via epigenetic reprogramming of monocytes. Proceedings of the National Academy of Sciences of the United States of America, 109(43), 17537-17542. https://doi.org/10.1073/pnas.1202870109

Knuesel, I., Chicha, L., Britschgi, M., Schobel, S. A., Bodmer, M., Hellings, J. A., Toovey, S., \& Prinssen, E. P. (2014). Maternal immune activation and abnormal brain development across CNS disorders. In Nature Reviews Neurology (Vol. 10, Issue 11, pp. 643-660). 
Nature Publishing Group. https://doi.org/10.1038/nrneurol.2014.187

1091

1092

1093

1094

1095

1096

1097

1098

1099

1100

1101

1102

1103

1104

1105

1106

1107

1108

1109

1110

1111

1112

1113

1114

1115

1116

1117

1118

1119

1120

Kolde, R. (2019). pheatmap: Pretty Heatmaps. R package version 1.0.12.

Kouzarides, T. (2007). Chromatin Modifications and Their Function. In Cell (Vol. 128, Issue 4, pp. 693-705). https://doi.org/10.1016/j.cell.2007.02.005

Kracht, L., Borggrewe, M., Eskandar, S., Brouwer, N., Chuva de Sousa Lopes, S. M., Laman, J. D., Scherjon, S. A., Prins, J. R., Kooistra, S. M., \& Eggen, B. J. L. (2020). Human fetal microglia acquire homeostatic immune-sensing properties early in development. Science, 369(6503), 530-537. https://doi.org/10.1126/science.aba5906

Krasemann, S., Madore, C., Cialic, R., Baufeld, C., Calcagno, N., El Fatimy, R., Beckers, L., O'Loughlin, E., Xu, Y., Fanek, Z., Greco, D. J., Smith, S. T., Tweet, G., Humulock, Z., Zrzavy, T., Conde-Sanroman, P., Gacias, M., Weng, Z., Chen, H., ... Butovsky, O. (2017). The TREM2-APOE Pathway Drives the Transcriptional Phenotype of Dysfunctional Microglia in Neurodegenerative Diseases. Immunity, 47(3), 566-581.e9. https://doi.org/10.1016/j.immuni.2017.08.008

Langmead, B., \& Salzberg, S. L. (2012). Fast gapped-read alignment with Bowtie 2. Nature Methods, 9(4), 357-359. https://doi.org/10.1038/nmeth.1923

Lavin, Y., Winter, D., Blecher-Gonen, R., David, E., Keren-Shaul, H., Merad, M., Jung, S., \& Amit, I. (2014). Tissue-resident macrophage enhancer landscapes are shaped by the local microenvironment. Cell, 159(6), 1312-1326. https://doi.org/10.1016/j.cell.2014.11.018

Leek, J. T., Johnson, W. E., Parker, H. S., Fertig, E. J., Jaffe, A. E., Zhang, Y., Storey, J. D., \& Collado, L. (2020). sva: Surrogate Variable Analysis. $R$ package version 3.36.0.

Li, Q., Cheng, Z., Zhou, L., Darmanis, S., Neff, N. F., Okamoto, J., Gulati, G., Bennett, M. L., Sun, L. O., Clarke, L. E., Marschallinger, J., Yu, G., Quake, S. R., Wyss-Coray, T., \& Barres, B. A. (2019). Developmental Heterogeneity of Microglia and Brain Myeloid Cells Revealed by Deep Single-Cell RNA Sequencing. Neuron, 101(2), 207-223.e10. https://doi.org/10.1016/j.neuron.2018.12.006

Liao, Y., Smyth, G. K., \& Shi, W. (2014). featureCounts: an efficient general purpose program for assigning sequence reads to genomic features. Bioinformatics, 30(7), 923930. https://doi.org/10.1093/bioinformatics/btt656

Longhi, L., Gesuete, R., Perego, C., Ortolano, F., Sacchi, N., Villa, P., Stocchetti, N., \& De 
Simoni, M. G. (2011). Long-lasting protection in brain trauma by endotoxin preconditioning. Journal of Cerebral Blood Flow and Metabolism, 31(9), 1919-1929. https://doi.org/10.1038/jcbfm.2011.42

Massagué, J., Seoane, J., \& Wotton, D. (2005). Smad transcription factors. Genes and Development, 19(23), 2783-2810. https://doi.org/10.1101/gad.1350705

Matcovitch-Natan, O., Winter, D. R., Giladi, A., Aguilar, S. V., Spinrad, A., Sarrazin, S., BenYehuda, H., David, E., González, F. Z., Perrin, P., Keren-Shaul, H., Gury, M., LaraAstaiso, D., Thaiss, C. A., Cohen, M., Halpern, K. B., Baruch, K., Deczkowska, A., Lorenzo-Vivas, E., ... Amit, I. (2016). Microglia development follows a stepwise program to regulate brain homeostasis. Science, 353(6301), aad8670. https://doi.org/10.1126/science.aad8670

Matt, S. M., Lawson, M. A., \& Johnson, R. W. (2016). Aging and peripheral lipopolysaccharide can modulate epigenetic regulators and decrease IL-1 $\beta$ promoter DNA methylation in microglia. Neurobiology of Aging, 47, 1-9. https://doi.org/10.1016/j.neurobiolaging.2016.07.006

Neher, J. J., \& Cunningham, C. (2019). Priming Microglia for Innate Immune Memory in the Brain. In Trends in Immunology (Vol. 40, Issue 4, pp. 358-374). Elsevier Ltd. https://doi.org/10.1016/j.it.2019.02.001

Netea, M. G. (2013). Training innate immunity: The changing concept of immunological memory in innate host defence. In European Journal of Clinical Investigation (Vol. 43, Issue 8, pp. 881-884). Eur J Clin Invest. https://doi.org/10.1111/eci.12132

Netea, M. G., Joosten, L. A. B., Latz, E., Mills, K. H. G., Natoli, G., Stunnenberg, H. G., O'Neill, L. A. J., \& Xavier, R. J. (2016). Trained immunity: A program of innate immune memory in health and disease. In Science (Vol. 352, Issue 6284, p. 427). American Association for the Advancement of Science. https://doi.org/10.1126/science.aaf1098

Netea, M. G., Quintin, J., \& Van Der Meer, J. W. M. (2011). Trained immunity: A memory for innate host defense. In Cell Host and Microbe (Vol. 9, Issue 5, pp. 355-361). https://doi.org/10.1016/j.chom.2011.04.006

Norden, D. M., Muccigrosso, M. M., \& Godbout, J. P. (2015). Microglial priming and enhanced reactivity to secondary insult in aging, and traumatic CNS injury, and neurodegenerative disease. In Neuropharmacology (Vol. 96, Issue PA, pp. 29-41). Elsevier Ltd. https://doi.org/10.1016/j.neuropharm.2014.10.028 
Nott, A., Holtman, I. R., Coufal, N. G., Schlachetzki, J. C. M., Yu, M., Hu, R., Han, C. Z., Pena, M., Xiao, J., Wu, Y., Keulen, Z., Pasillas, M. P., O'connor, C., Nickl, C. K., Schafer, S. T., Shen, Z., Rissman, R. A., Brewer, J. B., Gosselin, D., .. Glass, C. K. (2020). Brain cell type-specific enhancer-promoter interactome maps and disease-risk association. http://science.sciencemag.org/

Novakovic, B., Habibi, E., Wang, S. Y., Arts, R. J. W., Davar, R., Megchelenbrink, W., Kim, B., Kuznetsova, T., Kox, M., Zwaag, J., Matarese, F., van Heeringen, S. J., JanssenMegens, E. M., Sharifi, N., Wang, C., Keramati, F., Schoonenberg, V., Flicek, P., Clarke, L., ... Stunnenberg, H. G. (2016). $\beta$-Glucan Reverses the Epigenetic State of LPS-Induced Immunological Tolerance. Cell, 167(5), 1354-1368.e14. https://doi.org/10.1016/j.cell.2016.09.034

Oeckinghaus, A., \& Ghosh, S. (2009). The NF-kappaB family of transcription factors and its regulation. In Cold Spring Harbor perspectives in biology (Vol. 1, Issue 4). Cold Spring Harb Perspect Biol. https://doi.org/10.1101/cshperspect.a000034

Pal, R., Janz, M., Galson, D. L., Gries, M., Li, S., Jöhrens, K., Anagnostopoulos, I., Dörken, B., Mapara, M. Y., Borghesi, L., Kardava, L., David Roodman, G., Milcarek, C., \& Lentzsch, S. (2009). C/EBP $\beta$ regulates transcription factors critical for proliferation and survival of multiple myeloma cells. Blood, 114(18), 3890-3898. https://doi.org/10.1182/blood-2009-01-201111

Pardon, M. C. (2015). Lipopolysaccharide hyporesponsiveness: Protective or damaging response to the brain? In Romanian Journal of Morphology and Embryology (Vol. 56, Issue 3, pp. 903-913). Editura Academiei Romane. https://pubmed.ncbi.nlm.nih.gov/26662122/

Perkins, D. J., Patel, M. C., Blanco, J. C. G., \& Vogel, S. N. (2016). Epigenetic mechanisms governing innate inflammatory responses. Journal of Interferon and Cytokine Research, 36(7), 454-461. https://doi.org/10.1089/jir.2016.0003

Perry, V. H., \& Holmes, C. (2014). Microglial priming in neurodegenerative disease. Nature Reviews Neurology, 10(4), 217-224. https://doi.org/10.1038/nrneurol.2014.38

"Picard Toolkit." (2019). Broad Institute, GitHub Repository. http:///broadinstitute.github.io/picard/; Broad Institute.

Prinz, M., Erny, D., \& Hagemeyer, N. (2017). Ontogeny and homeostasis of CNS myeloid cells. In Nature Immunology (Vol. 18, Issue 4, pp. 385-392). Nature Publishing Group. https://doi.org/10.1038/ni.3703 
Quintin, J., Saeed, S., Martens, J. H. A., Giamarellos-Bourboulis, E. J., Ifrim, D. C., Logie, C., Jacobs, L., Jansen, T., Kullberg, B. J., Wijmenga, C., Joosten, L. A. B., Xavier, R. J., Van Der Meer, J. W. M., Stunnenberg, H. G., \& Netea, M. G. (2012). Candida albicans infection affords protection against reinfection via functional reprogramming of monocytes. Cell Host and Microbe, 12(2), 223-232. https://doi.org/10.1016/j.chom.2012.06.006

Raj, D. D. A., Jaarsma, D., Holtman, I. R., Olah, M., Ferreira, F. M., Schaafsma, W., Brouwer, N., Meijer, M. M., De Waard, M. C., Van der Pluijm, I., Brandt, R., Kreft, K. L., Laman, J. D., De Haan, G., Biber, K. P. H., Hoeijmakers, J. H. J., Eggen, B. J. L., \& Boddeke, H. W. G. M. (2014). Priming of microglia in a DNA-repair deficient model of accelerated aging. Neurobiology of Aging, 35(9), 2147-2160. https://doi.org/10.1016/j.neurobiolaging.2014.03.025

Raj, T., Rothamel, K., Mostafavi, S., Ye, C., Lee, M. N., Replogle, J. M., Feng, T., Lee, M., Asinovski, N., Frohlich, I., Imboywa, S., Von Korff, A., Okada, Y., Patsopoulos, N. A., Davis, S., McCabe, C., Paik, H. II, Srivastava, G. P., Raychaudhuri, S., ... De Jager, P. L. (2014). Polarization of the effects of autoimmune and neurodegenerative risk alleles in leukocytes. Science, 344(6183), 519-523. https://doi.org/10.1126/science.1249547

Ritchie, M. E., Phipson, B., Wu, D., Hu, Y., Law, C. W., Shi, W., \& Smyth, G. K. (2015). Limma powers differential expression analyses for RNA-sequencing and microarray studies. Nucleic Acids Research, 43(7), e47. https://doi.org/10.1093/nar/gkv007

Roach, J. C., Smith, K. D., Strobe, K. L., Nissen, S. M., Haudenschild, C. D., Zhou, D., Vasicek, T. J., Held, G. A., Stolovitzky, G. A., Hood, L. E., \& Aderem, A. (2007). Transcription factor expression in lipopolysaccharide-activated peripheral-blood-derived mononuclear cells. Proceedings of the National Academy of Sciences of the United States of America, 104(41), 16245-16250. https://doi.org/10.1073/pnas.0707757104

Robinson, M. D., McCarthy, D. J., \& Smyth, G. K. (2009). edgeR: A Bioconductor package for differential expression analysis of digital gene expression data. Bioinformatics, 26(1), 139-140. https://doi.org/10.1093/bioinformatics/btp616

Saeed, S., Quintin, J., Kerstens, H. H. D., Rao, N. A., Aghajanirefah, A., Matarese, F., Cheng, S. C., Ratter, J., Berentsem, K., Van Der Ent, M. A., Sharifi, N., JamssernMegens, E. M., Ter Huurne, M., Mandoli, A., Van Schaik, T., Ng, A., Burden, F., Downes, K., Frontini, M., ... Stunnenberg, H. G. (2014). Epigenetic programming of monocyte-to-macrophage differentiation and trained innate immunity. Science, 345(6204). https://doi.org/10.1126/science.1251086 
Salter, M. W., \& Stevens, B. (2017). Microglia emerge as central players in brain disease. In Nature Medicine (Vol. 23, Issue 9, pp. 1018-1027). Nature Publishing Group. https://doi.org/10.1038/nm.4397

Sarlus, H., \& Heneka, M. T. (2017). Microglia in Alzheimer's disease. In Journal of Clinical Investigation (Vol. 127, Issue 9, pp. 3240-3249). American Society for Clinical Investigation. https://doi.org/10.1172/JCI90606

Schaafsma, W, Zhang, X., van Zomeren, K. C., Jacobs, S., Georgieva, P. B., Wolf, S. A., Kettenmann, H., Janova, H., Saiepour, N., Hanisch, U. K., Meerlo, P., van den Elsen, P. J., Brouwer, N., Boddeke, H. W. G. M., \& Eggen, B. J. L. (2015). Long-lasting proinflammatory suppression of microglia by LPS-preconditioning is mediated by RelBdependent epigenetic silencing. Brain, Behavior, and Immunity, 48, 205-221. https://doi.org/10.1016/j.bbi.2015.03.013

Schaafsma, Wandert, Basterra, L. B., Jacobs, S., Brouwer, N., Meerlo, P., Schaafsma, A., Boddeke, E. W. G. M., \& Eggen, B. J. L. (2017). Maternal inflammation induces immune activation of fetal microglia and leads to disrupted microglia immune responses, behavior, and learning performance in adulthood. Neurobiology of Disease, 106, $291-$ 300. https://doi.org/10.1016/j.nbd.2017.07.017

Seeley, J. J., \& Ghosh, S. (2017). Molecular mechanisms of innate memory and tolerance to LPS. J. Leukoc. Biol, 101, 107-119. https://doi.org/10.1189/jlb.3MR0316-118RR

Semmler, A., Widmann, C. N., Okulla, T., Urbach, H., Kaiser, M., Widman, G., Mormann, F., Weide, J., Fliessbach, K., Hoeft, A., Jessen, F., Putensen, C., \& Heneka, M. T. (2013). Persistent cognitive impairment, hippocampal atrophy and EEG changes in sepsis survivors. Journal of Neurology, Neurosurgery and Psychiatry, 84(1), 62-70. https://doi.org/10.1136/jnnp-2012-302883

Shao, Z., Zhang, Y., Yuan, G. C., Orkin, S. H., \& Waxman, D. J. (2012). MAnorm: A robust model for quantitative comparison of ChIP-Seq data sets. Genome Biology, 13(3), R16. https://doi.org/10.1186/gb-2012-13-3-r16

Sierra, A., Gottfried-Blackmore, A. C., Mcewen, B. S., \& Bulloch, K. (2007). Microglia derived from aging mice exhibit an altered inflammatory profile. GLIA, 55(4), 412-424. https://doi.org/10.1002/glia.20468

Simpson-Abelson, M. R., Hernandez-Mir, G., Childs, E. E., Cruz, J. A., Poholek, A. C., Chattopadhyay, A., Gaffen, S. L., \& McGeachy, M. J. (2017). CCAAT/Enhancer-binding protein $\beta$ promotes pathogenesis of EAE. Cytokine, 92, 24-32. 
https://doi.org/10.1016/j.cyto.2017.01.005

1254

1255

1256

1257

1258

1259

1260

1261

1262

1263

1264

1265

1266

1267

1268

1269

1270

1271

1272

1273

1274

1275

1276

1277

1278

1279

1280

1281

1282

1283

1284

1285

Straccia, M., Gresa-Arribas, N., Dentesano, G., Ejarque-Ortiz, A., Tusell, J. M., Serratosa, J., Solà, C., \& Saura, J. (2011). Pro-inflammatory gene expression and neurotoxic effects of activated microglia are attenuated by absence of CCAAT/enhancer binding protein $\beta$. Journal of Neuroinflammation, 8, 156. https://doi.org/10.1186/1742-2094-8-156

Tay, T. L., Mai, D., Dautzenberg, J., Fernández-Klett, F., Lin, G., Sagar, Datta, M., Drougard, A., Stempfl, T., Ardura-Fabregat, A., Staszewski, O., Margineanu, A., Sporbert, A., Steinmetz, L. M., Pospisilik, J. A., Jung, S., Priller, J., Grün, D., Ronneberger, O., \& Prinz, M. (2017). A new fate mapping system reveals context-dependent random or clonal expansion of microglia. Nature Neuroscience, April. https://doi.org/10.1038/nn.4547

Valente, T., Straccia, M., Gresa-Arribas, N., Dentesano, G., Tusell, J. M., Serratosa, J., Mancera, P., Solà, C., \& Saura, J. (2013). CCAAT/enhancer binding protein $\delta$ regulates glial proinflammatory gene expression. Neurobiology of Aging, 34(9), 2110-2124. https://doi.org/10.1016/j.neurobiolaging.2013.02.007

Wang, J., Lee, S., Teh, C. E. Y., Bunting, K., Ma, L., \& Shannon, M. F. (2009). The transcription repressor, ZEB1, cooperates with CtBP2 and HDAC1 to suppress IL-2 gene activation in T cells. International Immunology, 21(3), 227-235.

https://doi.org/10.1093/intimm/dxn143

Warnes, G., Bolker, B., Bonebakker, L., Gentleman, R., Liaw, W., Lumley, T., Mächler, M., Magnússon, Á., Moeller, S., Schwartz, M., Venables, B., Huber, W., Liaw, A., Grégory, R., Warnes, B., Bonebakker, L., Liaw, W. H. A., Lumley, T., Mächler, M., ... Venables, B. (2015). gplots: Various R Programming Tools for Plotting Data. Undefined.

Weeda, G., Donker, I., De Wit, J., Morreau, H., Janssens, R., Vissers, C. J., Nigg, A., Van Steeg, H., Bootsma, D., \& Hoeijmakers, J. H. J. (1997). Disruption of mouse ERCC1 results in a novel repair syndrome with growth failure, nuclear abnormalities and senescence. Current Biology, 7(6), 427-439. https://doi.org/10.1016/S09609822(06)00190-4

Wendeln, A. C., Degenhardt, K., Kaurani, L., Gertig, M., Ulas, T., Jain, G., Wagner, J., Häsler, L. M., Wild, K., Skodras, A., Blank, T., Staszewski, O., Datta, M., Centeno, T. P., Capece, V., Islam, M. R., Kerimoglu, C., Staufenbiel, M., Schultze, J. L., ... Neher, J. J. (2018). Innate immune memory in the brain shapes neurological disease hallmarks. Nature, 556(7701), 332-338. https://doi.org/10.1038/s41586-018-0023-4 
Wickham, H., Chang, W., Henry, L., Pedersen, T., Takahashi, K., Wilke, C., Woo, K., Yutani, H., \& Dunnington, D. (2016). Welcome | ggplot2. Springer-Verlag New York. https://ggplot2-book.org/

Yeh, H., \& Ikezu, T. (2019). Transcriptional and Epigenetic Regulation of Microglia in Health and Disease. In Trends in Molecular Medicine (Vol. 25, Issue 2, pp. 96-111). Elsevier Ltd. https://doi.org/10.1016/j.molmed.2018.11.004

Yu, G., Wang, L. G., Han, Y., \& He, Q. Y. (2012). ClusterProfiler: An R package for comparing biological themes among gene clusters. OMICS A Journal of Integrative Biology, 16(5), 284-287. https://doi.org/10.1089/omi.2011.0118

Yu, G., Wang, L. G., \& He, Q. Y. (2015). ChIP seeker: An R/Bioconductor package for ChIP peak annotation, comparison and visualization. Bioinformatics, 31(14), 2382-2383. https://doi.org/10.1093/bioinformatics/btv145

Yu, J. T., Lee, C. H., Yoo, K. Y., Choi, J. H., Li, H., Park, O. K., Yan, B., Hwang, I. K., Kwon, Y. G., Kim, Y. M., \& Won, M. H. (2010). Maintenance of anti-inflammatory cytokines and reduction of glial activation in the ischemic hippocampal CA1 region preconditioned with lipopolysaccharide. Journal of the Neurological Sciences, 296(1-2), 69-78. https://doi.org/10.1016/j.jns.2010.06.004

Zhang, X., Heng, Y., Kooistra, S. M., van Weering, H. R. J., Brummer, M. L., Gerrits, E., Wesseling, E. M., Brouwer, N., Nijboer, T. W., Dubbelaar, M. L., Boddeke, E. W. G. M., \& Eggen, B. J. L. (2020). Intrinsic DNA damage repair deficiency results in progressive microglia loss and replacement. GLIA. https://doi.org/10.1002/glia.23925

Zhou, Q., Liu, M., Xia, X., Gong, T., Feng, J., Liu, W., Liu, Y., Zhen, B., Wang, Y., Ding, C., \& Qin, J. (2017). A mouse tissue transcription factor atlas. Nature Communications, 8. https://doi.org/10.1038/ncomms15089

Zrzavy, T., Hametner, S., Wimmer, I., Butovsky, O., Weiner, H. L., \& Lassmann, H. (2017). Loss of "homeostatic" microglia and patterns of their activation in active multiple sclerosis. Brain, 140(7), 1900-1913. https://doi.org/10.1093/brain/awx113 